\title{
Targeted Genome Editing of Bacteria Within Microbial
}

\section{Communities}

4 Benjamin E. Rubin ${ }^{1,2,13}$, Spencer Diamond ${ }^{1,3,13}$, Brady F. Cress ${ }^{1,2,13}$, Alexander Crits-Christoph ${ }^{4}$, 5 Christine $\mathrm{He}^{1,2,3}$, Michael Xu $\mathrm{u}^{1,2}$, Zeyi Zhou ${ }^{1,2}$, Dylan C. Smock ${ }^{1,2}$, Kimberly Tang ${ }^{1,2}$, Trenton $\mathrm{K}$.

6 Owens $^{5}$, Netravathi Krishnappa ${ }^{1}$, Rohan Sachdeva ${ }^{1,3}$, Adam M. Deutschbauer ${ }^{4,5}$, Jillian F.

7 Banfield ${ }^{1,3,6,7 *} \&$ Jennifer A. Doudna ${ }^{1,2,8-12 *}$

$8{ }^{1}$ Innovative Genomics Institute, University of California, Berkeley, CA, USA. ${ }^{2}$ Department of Molecular and 9 Cell Biology, University of California, Berkeley, CA, USA. ${ }^{3}$ Department of Earth and Planetary Science, 10 University of California, Berkeley, CA, USA. ${ }^{4}$ Department of Plant and Microbial Biology, University of 11 California, Berkeley, CA, USA. ${ }^{5}$ Environmental Genomics and Systems Biology Division, Lawrence 12 Berkeley National Laboratory, Berkeley, California, USA. ${ }^{6}$ Environmental Science, Policy and Management, 13 University of California, Berkeley, CA, USA. ${ }^{7}$ School of Earth Sciences, University of Melbourne, 14 Melbourne, Victoria, Australia. ${ }^{8}$ California Institute for Quantitative Biosciences, University of California, 15 Berkeley, CA, USA. ${ }^{9}$ Department of Chemistry, University of California, Berkeley, CA, USA. ${ }^{10}$ Howard 16 Hughes Medical Institute, University of California, Berkeley, CA, USA. ${ }^{11}$ Molecular Biophysics \& Integrated 17 Bioimaging Division, Lawrence Berkeley National Laboratory, Berkeley, CA, USA ${ }^{12}$ Gladstone Institutes, 18 University of California, San Francisco, CA, USA. ${ }^{13}$ These authors contributed equally to this work: 19 Benjamin E. Rubin, Spencer Diamond, Brady F. Cress.

20

21 *e-mail: doudna@berkeley.edu; jbanfield@berkeley.edu 
22 Knowledge of microbial gene functions comes from manipulating the DNA of individual

23 species in isolation from their natural communities. While this approach to microbial

24 genetics has been foundational, its requirement for culturable microorganisms has left the

25 majority of microbes and their interactions genetically unexplored. Here we describe a

26 generalizable methodology for editing the genomes of specific organisms within a

27 complex microbial community. First, we identified genetically tractable bacteria within a

28 community using a new approach, Environmental Transformation Sequencing (ET-Seq),

29 in which non-targeted transposon integrations were mapped and quantified following

30 community delivery. ET-Seq was repeated with multiple delivery strategies for both a nine-

31 member synthetic bacterial community and a $\mathbf{2 0 0 - m e m b e r ~ m i c r o b i a l ~ b i o r e m e d i a t i o n ~}$

32 community. We achieved insertions in 10 species not previously isolated and identified

33 natural competence for foreign DNA integration that depends on the presence of the

34 community. Second, we developed and used DNA-editing All-in-one RNA-guided CRISPR-

35 Cas Transposase (DART) systems for targeted DNA insertion into organisms identified as

36 tractable by ET-Seq, enabling organism- and locus-specific genetic manipulation within

37 the community context. These results demonstrate a strategy for targeted genome editing

38 of specific organisms within microbial communities, establishing a new paradigm for

39 microbial manipulation relevant to research and applications in human, environmental,

40 and industrial microbiomes.

42 Genetic mutation and observation of phenotypic outcomes are the primary means of deciphering

43 gene function in microorganisms. This classical genetic approach requires manipulation of

44 isolated species, limiting knowledge in three fundamental ways. First, the vast majority of

45 microorganisms have not been isolated in the laboratory and are thus largely untouched by

46 molecular genetics ${ }^{1}$. Second, emergent properties of microbial communities that may arise due

47 to interactions between their constituents, remain mostly unexplored ${ }^{2}$. Third, microorganisms 
48 grown and studied in isolation quickly adapt to their new lab environment, obscuring their true

49 "wild type" physiology 3 . Since most microorganisms relevant to the environment, industry and

50 health live in communities, approaches for precision genome modification (editing) in community

51 contexts will be transformative.

52 Advances toward genome editing within microbial communities have included assessing

53 gene transfer to microbiomes using selectable markers ${ }^{4-9}$, microbiome manipulation leveraging

54 pre-modified isolates ${ }^{10}$, and use of temperate phage for species-specific integration of genetic

55 payloads ${ }^{11}$. However, a generalizable strategy for programmable organism- and locus-specific

56 editing within a community of wild-type microbes has not yet been reported ${ }^{12}$.

57 Here we show that specific organisms within microbial communities can be targeted for

58 site-specific genome editing, enabling manipulation of species without requiring prior isolation or

59 engineering. Using a new method developed for this study, Environmental Transformation

60 Sequencing (ET-Seq), we identified genetically accessible species within a nine-member

61 synthetic community and among previously non-isolated species in a 197-member bioremediation

62 community. These results enabled targeted genome editing of microbes in the nine-member

63 community using DNA-editing All-in-one RNA-guided CRISPR-Cas Transposase (DART)

64 systems developed here. The resulting species-specific editing provides the first broadly

65 applicable strategy for organism- and locus-specific genetic manipulation within a microbial

66 community, hinting at new emergent properties of member organisms and methods for controlling

67 microorganisms within their native environments.

69 ET-Seq detects genetically accessible microbial community members

70 Editing organisms within a complex microbiome requires knowing which constituents are

71 accessible to nucleic acid delivery and editing. We developed ET-Seq to assess the ability of

72 individual species within a microbial community to acquire and integrate exogenous DNA (Fig.

73 1a). In ET-Seq, a microbial community is exposed to a randomly integrating mobile genetic 
74 element (here, a mariner transposon), and in the absence of any selection, total community DNA

75 is then extracted and sequenced using two protocols. In the first, we enrich and sequence the

76 junctions between the inserted and host DNA to determine insertion location and quantity in each

77 host. This step requires comparison of the junctions to previously sequenced community

78 reference genomes. In the second, we conduct low-depth metagenomic sequencing to quantify

79 the abundance of each community member in a sample (Extended Data Fig. 1a). Together these

80 sequencing procedures provide relative insertion efficiencies for microbiome members. To

81 normalize these data according to a known internal standard, we add to every sample a uniform

82 amount of genomic DNA from an organism that has previously been transformed with, and

83 selected for, a mariner transposon. In the internal standard, we expect every genome to contain

84 an insertion. The final output of ET-Seq is a fractional number representing the proportion of a

85 target organism's population that harbored transposon insertions at the time DNA was extracted,

86 or insertion efficiency (Extended Data Fig. 1b). To facilitate the analysis of these disparate data,

87 we developed a complete bioinformatic pipeline for quantifying insertions and normalizing results

88 according to both the internal control and metagenomic abundance

89 (https://github.com/SDmetagenomics/ETsuite and Methods). Together the experimental and

90 bioinformatic approaches of ET-Seq reveal species-specific genetic accessibility by measuring

91 the percentage of each member of a given microbiome that acquires a transposon insertion. 

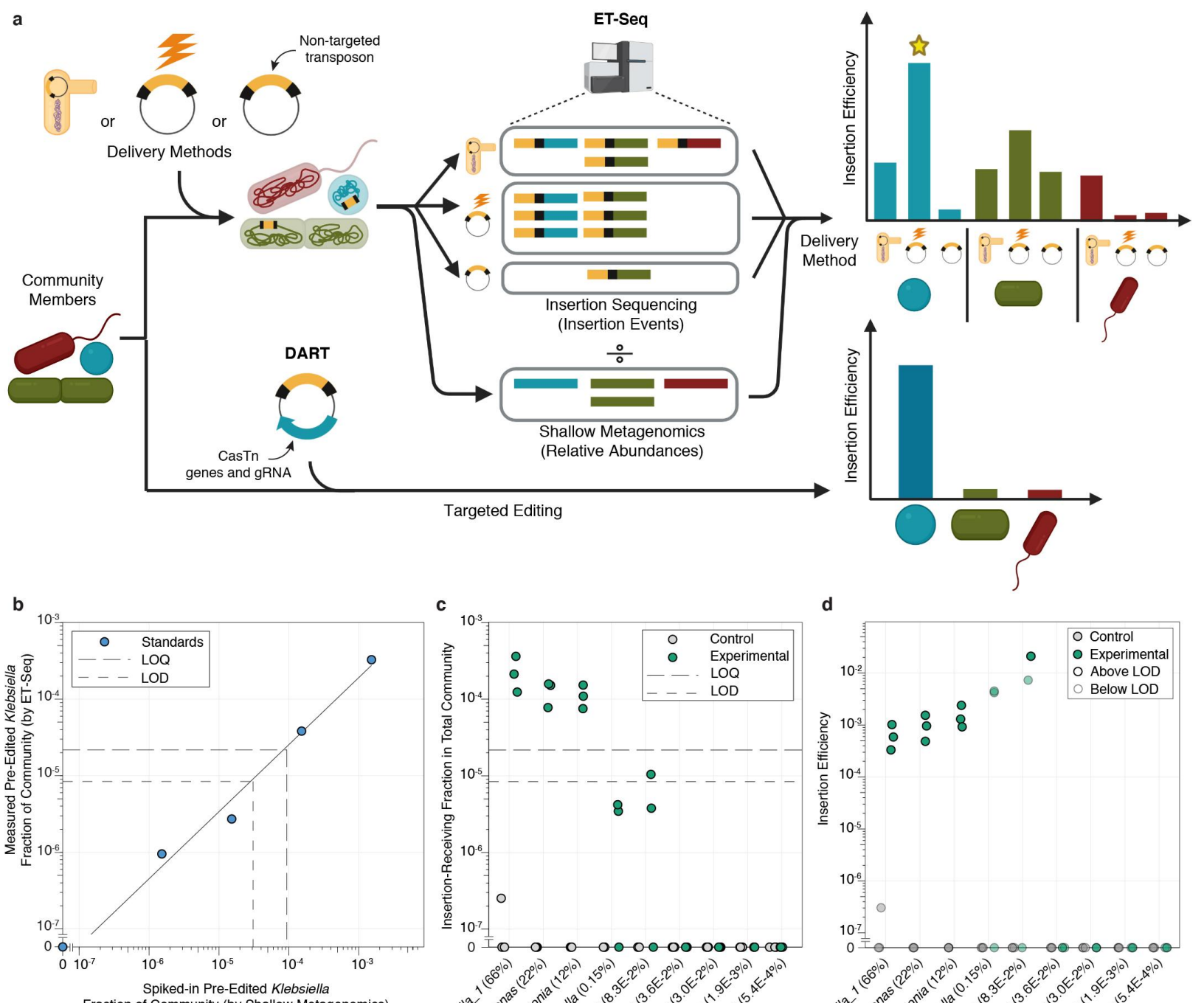

c
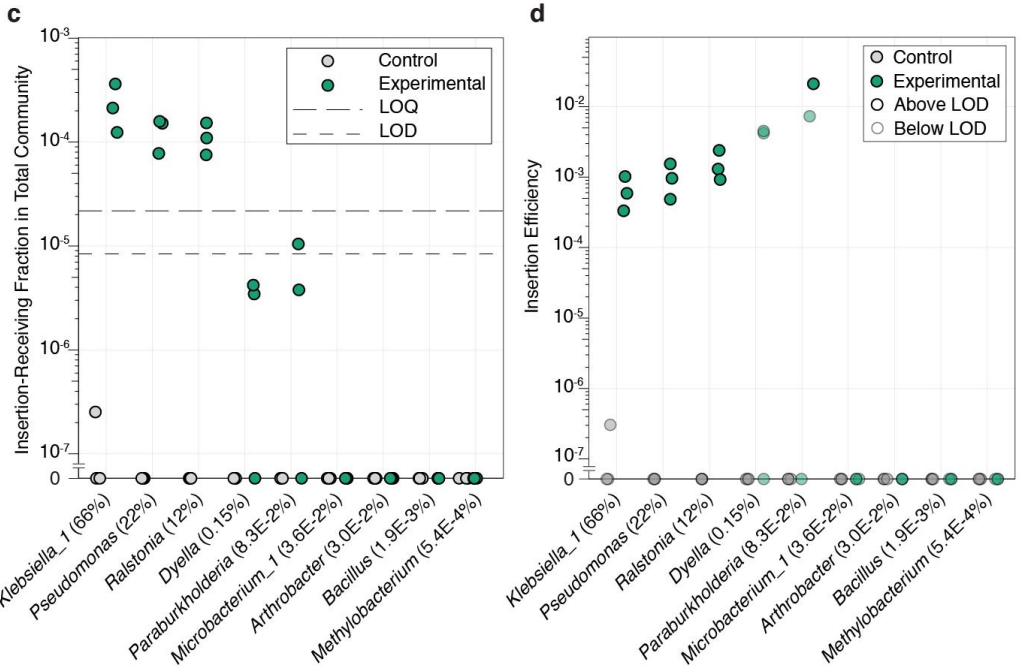

Fig. 1 | ET-Seq for quantitative measurement of insertion efficiency in a microbial community. a, ET-

Seq provides data on insertion efficiency of multiple delivery approaches, including conjugation, electroporation, and natural DNA transformation, on microbial community members. In this illustrative example, the blue strain is most amenable to electroporation (star). This data allows for the determination of feasible targets and delivery methods for DART targeted editing. b, ET-Seq determined efficiencies for known quantities of spiked-in pre-edited K. michiganensis (Klebsiella_1). Data shown is the mean of three technical replicates. LOD is the lowest insertion fraction at which accurate detection of insertions is expected and LOQ is the lower limit at which this fraction is expected to be quantifiable. Solid line is the fit of the linear regression to the data not including zero ( $n=4$ independent samples) that is used to calculate 
LOD and LOQ $\left(\right.$ slope $=0.2137$; intercept $\left.=1.813^{*} 10^{-6}\right) . \mathbf{c}-\mathrm{d}$, ET-Seq determined insertion efficiencies in the

104 nine-member consortium ( $n=3$ biological replicates) with conjugative delivery shown as $\mathbf{c}$, a portion of the

105 entire community and $\mathbf{d}$, a portion of each species. Control samples received no exogenous DNA. Average

106 relative abundances of community constituents across conjugation samples ( $n=6$ independent samples)

107 are indicated in parentheses. LOD and LOQ are indicated in plots by short and long dashed lines

108 respectively.

ET-Seq was developed and tested on a nine-member microbial consortium made up of

111 bacteria from three phyla that are often detected and play important metabolic roles within soil

112 microbial communities (Supplementary Table 1). We initially endeavored to test the accuracy and

113 detection limit by adding to the nine-member community a known amount of a previously prepared

114 mariner transposon library of one of its member species, Klebsiella michiganensis M5a1

115 (Klebsiella_1). The ET-Seq derived insertion efficiencies were closely correlated to the known

116 fractions of edited K. michiganensis present in each sample (Fig. 1b). Using this data we

117 calculated a limit of detection (LOD) and limit of quantification (LOQ) for our approach (Methods).

118 The LOD suggests that a fraction of $\geq 8.4^{*} 10^{-6}$ of transformed cells out of the total community

119 would be detectable by ET-Seq.

120 Next, the mariner transposon vector was delivered to the nine-member community through

121 conjugation. We could measure conjugation reproducibly and quantitatively in the three species

122 that grew to make up over $99 \%$ of the community (Fig. 1c). We further normalized insertion

123 efficiency in each species according to its abundance so that their insertion efficiencies represent

124 insertion containing cells as a portion of total cells for each species (Fig. 1d). Even for

125 Paraburkholderia caledonica, which made up $\sim 0.1 \%$ of the community, we could measure

126 insertions. We detected no insertions in the remaining community members, which was expected

127 given their extreme rarity in the community (less than $0.05 \%$ ). 
We next used ET-Seq to compare insertion efficiencies in the nine-member community

129 after transposon delivery by conjugation, natural transformation with no induction of competence,

130 or electroporation of the transposon vector. Together these approaches showed reproducible

131 insertion efficiencies above the LOD in five of the nine community members (Fig. 2a and Extended

132 Data Fig. 2). Additionally we could identify preferred delivery methods for some members in this

133 community context, such as electroporation being consistently reproducible for Dyella japonica

134 UNC79MFTsu3.2 while conjugation was not. These results show that ET-Seq can identify and

135 quantify genetic manipulation of microbial community members and reveal suitable DNA delivery

136 methods for each. 

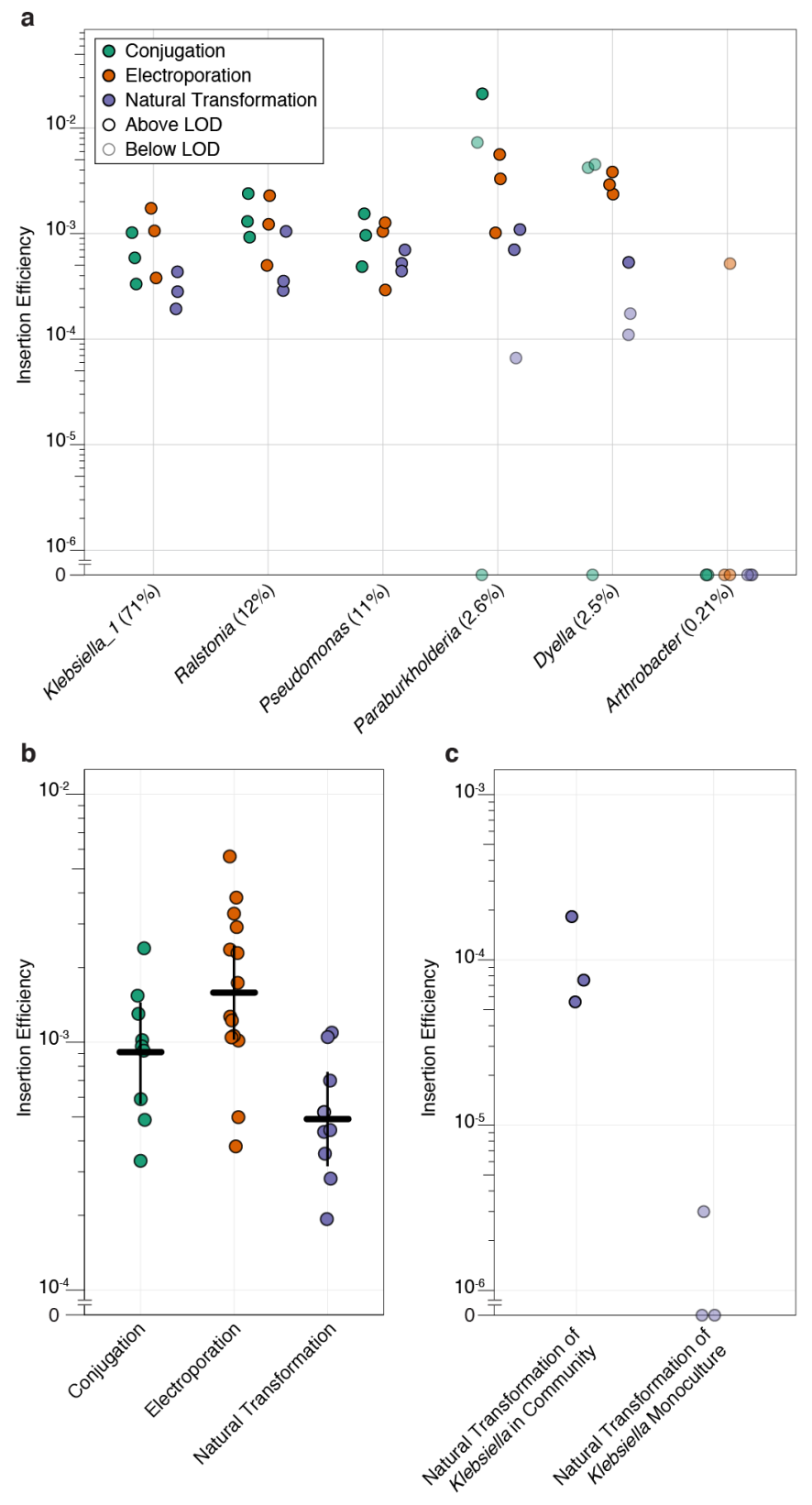

139 Fig. 2 | ET-Seq detection of insertion efficiency across multiple delivery approaches. a, ET-Seq

140 determined insertion efficiencies for conjugation, electroporation, and natural transformation on the nine-

141 member consortium ( $n=3$ biological replicates). Only members with at least one positive insertion efficiency

142 value across the delivery methods are shown. Average relative abundance across all samples $(n=18$

143 independent samples) is indicated in parentheses. b, Comparing delivery strategies across data from all

144 organisms. Cross bars indicate the mean value and whiskers denote the $95 \%$ confidence interval for the 145 mean (Conjugation $n=9$; Electroporation $n=14$; Natural Transformation $n=9$ ). c, Comparison of insertion 
146 efficiencies measured for natural transformation of $K$. michiganensis (Klebsiella_1) in isolated culture $(\mathrm{n}=$

1473 biological replicates) compared to K. michiganensis grown in the community context $(\mathrm{n}=3$ biological

148 replicates).

Notably, five organisms exhibited some degree of natural competency, although average

151 efficiencies were significantly lower for natural transformation than for delivery through

152 electroporation (ANOVA followed by Tukey's HSD; two-sided test; $p=0.0009$ ) (Fig. 2b). To the

153 best of our knowledge, no isolates of the Klebsiella genus including K. michiganensis are known

154 to be naturally competent. We conducted a second experiment to compare the insertion efficiency

155 of $K$. michiganensis cultivated in isolation versus grown in the community context. ET-Seq

156 returned no values above the LOD for natural transformation of $K$. michiganensis in isolation, but

157 within the community ET-Seq returned values well into the quantifiable range (Fig. 2c). This

158 apparent emergent property of natural competence within a small synthetic community provides

159 tantalizing support for the possibility of community induced natural transformation, an idea suggested

160 in previous work, but experimentally unstudied due to lack of tools for measuring horizontal gene

161 transfer events within a community ${ }^{13,14}$.

163 Genetic accessibility of uncultivated species within an environmental microbiome

164 To test the potential for editing in a complex and environmentally realistic community that has not

165 been reduced to isolates, we conducted ET-Seq on a genomically characterized 197 member

166 bioreactor-derived consortium that degrades thiocyanate $\left(\mathrm{SCN}^{-}\right)$, a toxic byproduct of gold

167 processing ${ }^{15}$. We sampled biofilm from the reactor and conducted ET-Seq with a panel of delivery

168 techniques: conjugation, electroporation, and natural transformation. Across ET-Seq replicates at

169 least one measurement above the LOD was identified for 15 members of the bioreactor

170 community. We also note that the transformed organisms make up $\sim 87 \%$ of the bacterial fraction

171 by relative abundance (Fig. 3a, Extended Data Fig. 3). Ten of these organisms were species that 
172 had not previously been isolated or edited (Supplementary Table 1), and overall members from 7

173 of the 12 phyla detected in this consortium were successfully transformed (Fig. 3b). This included

174 an Afipia sp. known to play an important role in the thiocyanate degradation process. Additionally,

175 one of the transposon recipients, Microbacterium ginsengisoli (Microbacterium_3), is a putative

176 host for Saccharibacteria, a candidate phyla radiation (CPR) organism that has been observed in

177 this reactor system ${ }^{16}$. Notably, members of the CPR are resistant to typical isolation techniques

178 due to heavy dependence on other community members, and little is known about the nature of

179 their likely symbiotic relationships with other organisms ${ }^{17}$. Here, ET-Seq has uncovered a

180 genetically tractable putative host organism, raising the possibility of genetically editing the host

181 to probe CPR/host symbiotic relationships within a complex microbial community. In this way, ET-

182 Seq reveals genetic accessibility and the tools necessary to achieve it in previously

183 unapproachable and biologically important members of an environmentally relevant community.
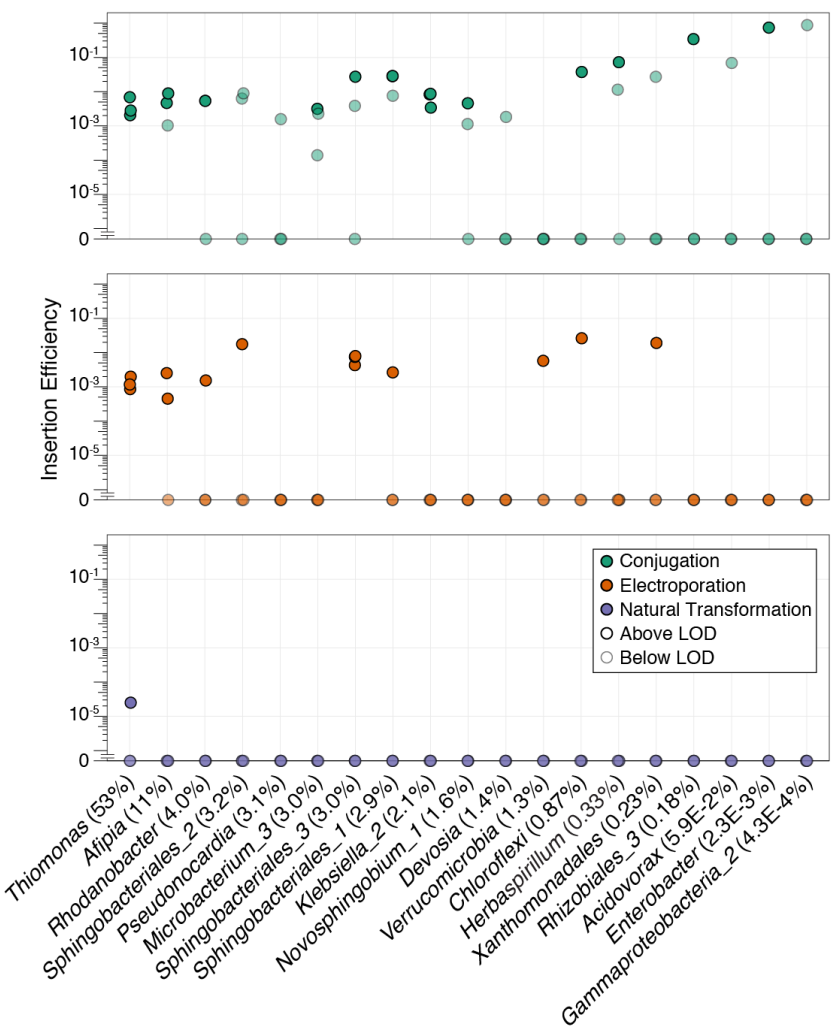

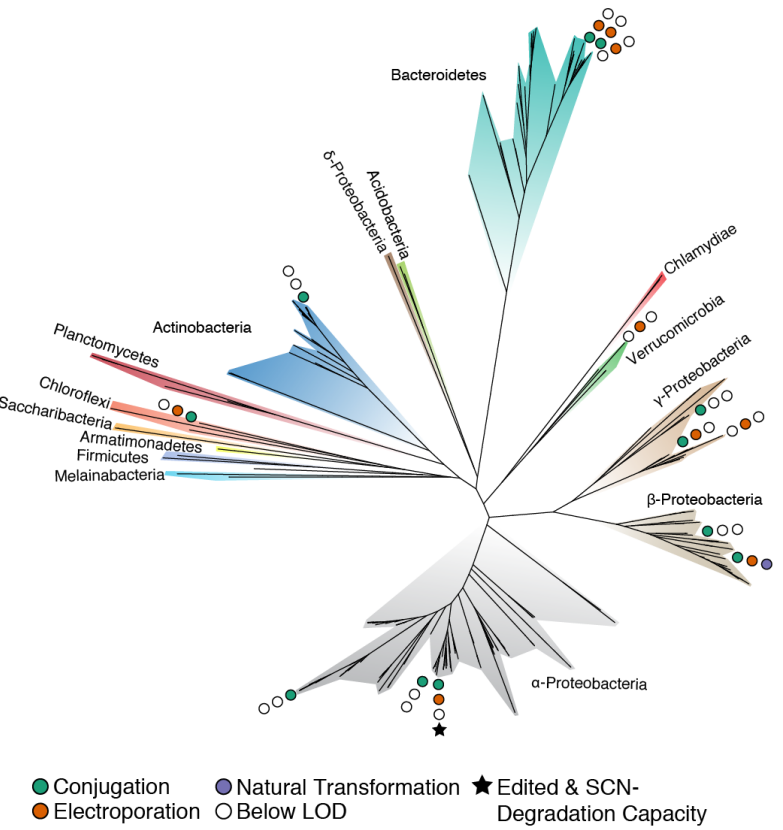

184

185

Fig. 3 | ET-Seq detection of insertion efficiency in thiocyanate-degrading bioreactor. a, ET-Seq 
determined insertion efficiencies for conjugation, electroporation, and natural transformation on the thiocyanate-degrading bioreactor community ( $n=3$ biological replicates). Average relative abundance across all samples is indicated in parentheses $(n=17$ independent samples). b, A ribosomal protein S3 (rpS3) phylogenetic tree of all organisms in the thiocyanate-degrading bioreactor genome database. Only community members receiving at least one insertion by conjugation, electroporation, or natural

191 transformation detectable above LOD are indicated by filled circles. Filled circles indicate success of 192 method and open circles indicate method was not detected. A star indicates genomically encoded SCN degradation capacity in organisms shown to be edited by at least one method. Tree was constructed from an alignment of 262 rps3 protein sequences using IQ-TREE.

\section{Targeted genome editing in microbial communities using CRISPR-Cas transposases}

197 The ability to introduce genome edits to a single type of organism in a microbial community and

198 to target those edits to a defined location within its genome would be a foundational advance in 199 microbiological research and would have many useful applications. We reasoned that RNA200 guided CRISPR-Cas Tn7 transposases could provide the ability to both ablate function of targeted 201 genes and deliver customized genetic cargo in organisms shown to be genetically tractable by 202 ET-Seq ${ }^{18-20}$ (Fig. 1a). However, the two-plasmid ShCasTn ${ }^{18}$ and three-plasmid VcCasTn ${ }^{19}$ 203 systems are not amenable to efficient delivery within complex microbial communities or even 204 beyond $E$. coli due to their multiple plasmids. Since ET-Seq identified conjugation and 205 electroporation as broadly effective delivery approaches in the tested communities, we designed 206 and constructed all-in-one conjugative versions of these CasTn vectors that could be used for 207 delivery by either strategy (Fig. 4a and Methods). These DART systems are barcoded and 208 compatible with the same sequencing methods used for ET-Seq, and can be used to assay the 209 efficacy of CRISPR-Cas-guided transposition into the genome of a target organism. 


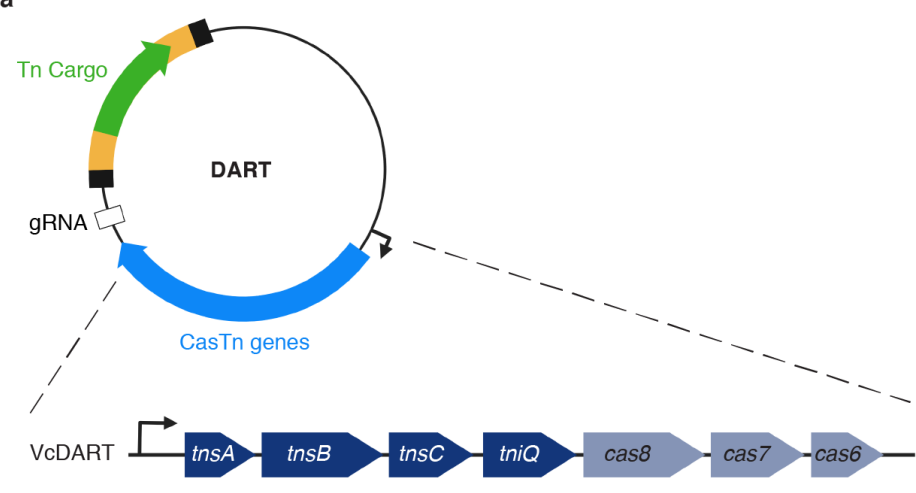

or

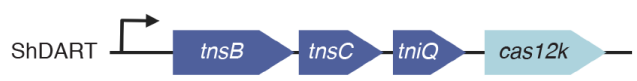

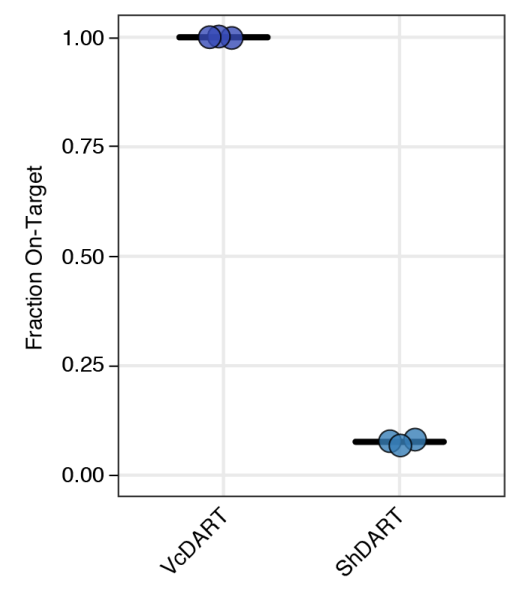

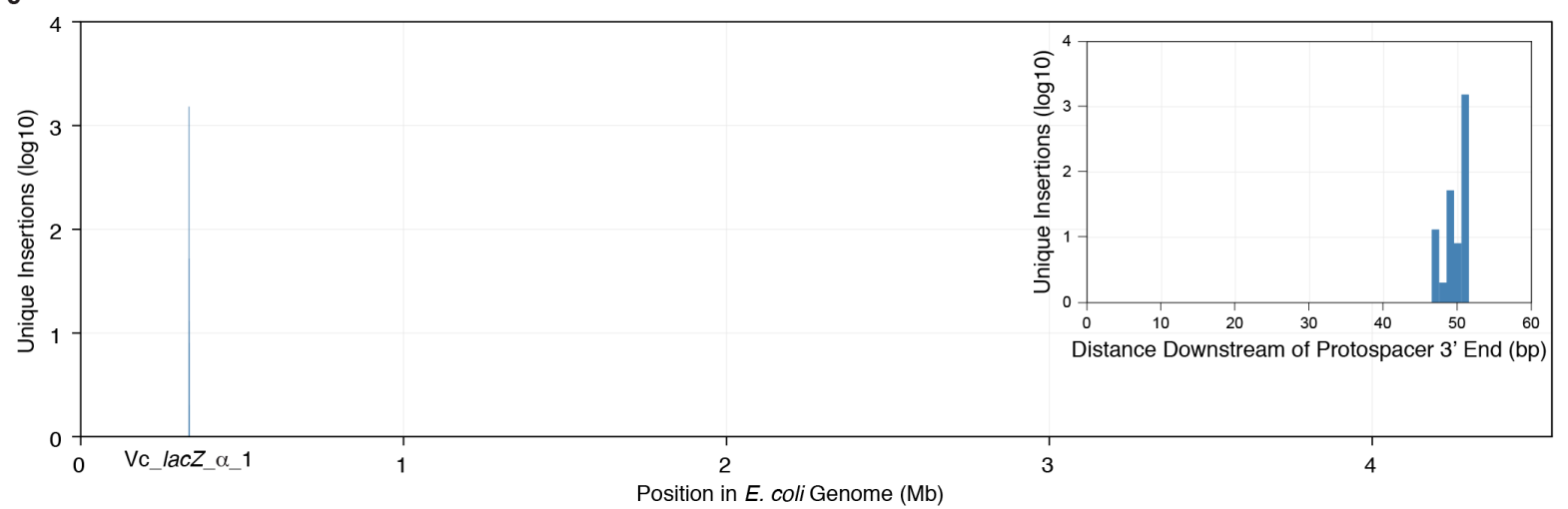

d

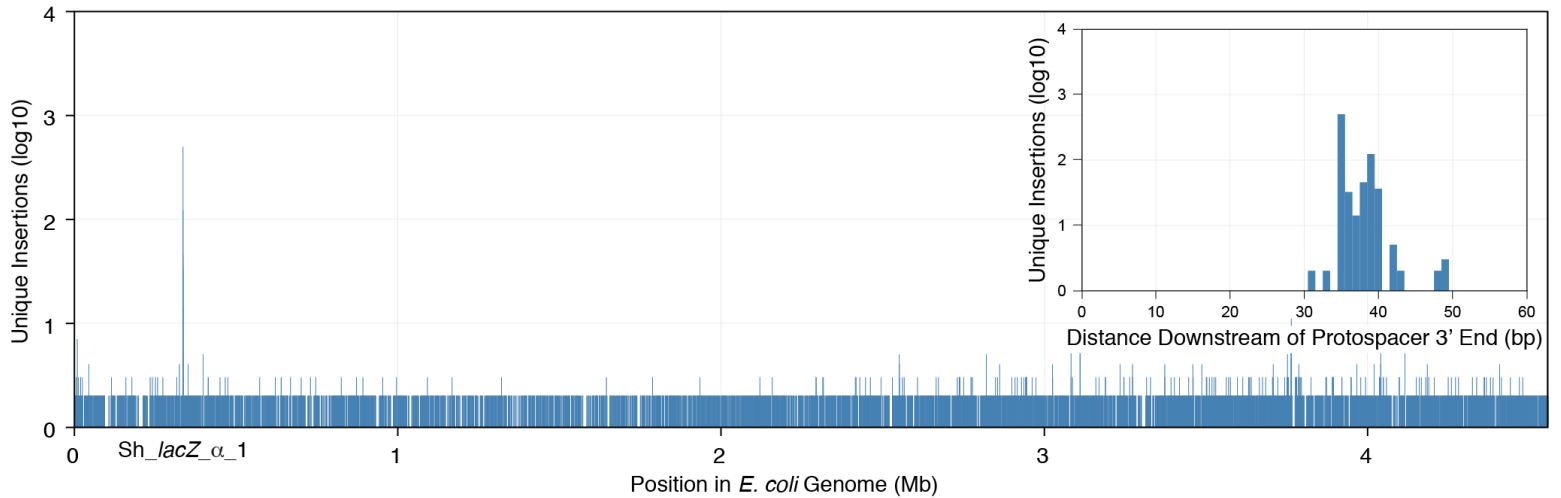

212 Fig. 4 | Benchmarking all-in-one conjugal targeted vectors. a, Schematic of VcDART and ShDART

213 delivery vectors. b, Fraction of insertions that occur in a $60 \mathrm{bp}$ window around the target site. Mean for

214 three independent biological replicates is shown as cross bars. c-d, Aggregate unique insertion counts (n

$215=3$ biological replicates) across the $E$. coli BL21(DE3) genome, determined by presence of unique barcodes,

216 using c, VcDART and d, ShDART. The inset shows a 60 bp window downstream of the target site. Insertion

217 distance downstream of the target site is calculated from the 3' end of the protospacer. 
We compared the transposition efficiency and specificity of the DART systems in E. coli

220 in order to select the most promising candidate for targeted genome editing in microbial

221 communities. VcDART and ShDART systems harboring $\mathrm{Gm}^{\mathrm{R}}$ cargo with a lacZ-targeting or non-

222 targeting guide RNA were conjugated into E. coli to quantify transposition efficiency, and target

223 site specificity was assayed using ET-Seq following outgrowth of transconjugants in selective

224 medium (Methods and Extended Data Fig. 4a). While ShDART yielded approximately tenfold

225 more colonies possessing insertions than ShDART (Extended Data Fig. 4), >92\% of the

226 selectable colonies obtained using ShDART were off-target, compared to no detectable off-target

227 insertions for VcDART (Fig. 4b-d). Due to VcDART's high target site specificity and the

228 undesirable propensity for ShCasTn to co-integrate its donor plasmid ${ }^{21,22}$, we focused on VcDART

229 to test the potential for targeted microbial community genome editing.

\section{Targeted microbial community editing by programmable transposition}

232 We reasoned that RNA-programmed transposition could be deployed for targeted editing of 233 specific types of organisms within a microbial consortium. ET-Seq had shown two species within

234 the nine-member community, K. michiganesis and Pseudomonas simiae WCS417, to be both

235 abundant and tractable by conjugation (Fig. 1d). We targeted both of these organisms using 236 conjugation to introduce the VcDART vector into the community with guide RNAs specific to their 237 genomes (Fig. 5a). Insertions were designed to produce loss-of-function mutations in the $K$. 238 michiganesis and $P$. simiae pyrF gene, an endogenous counterselectable marker allowing growth

239 in the presence of 5-fluoroorotic acid (5-FOA) when disrupted. The transposons carried two 240 antibiotic resistance markers conferring resistance to streptomycin and spectinomycin (aadA) and 241 carbenicillin (b/a). Together the simultaneous loss-of-function and gain-of-function mutations

242 allowed for a strong selective regime. VcDART targeted to $K$. michiganensis or to $P$. simiae pyrF 243 followed by selection led to enrichment of these organisms to $\sim 98 \%$ and $\sim 97 \%$ pure culture 
244 respectively (Fig. 5b). No outgrowth was detected when using a guide RNA that did not target

245 these respective microbial genomes. Recovered transformant colonies of $K$. michiganensis and

246 P. simiae analyzed by PCR and Sanger sequencing showed full length, pyrF-disrupting VcDART

247 transposon insertions $48-49$ bp downstream of the guide RNA target site, consistent with

248 CRISPR-Cas transposase-catalyzed transposition events at the desired genomic location (Fig.

$2494 \mathrm{c}$ and $5 \mathrm{c}$ ). These results demonstrate that targeted genome editing using DART enables genetic

250 manipulation of distinct members of a complex microbial community. This targeted editing of

251 microorganisms in a community context can also enable subsequent exploitation of modified

252 phenotypes.

253
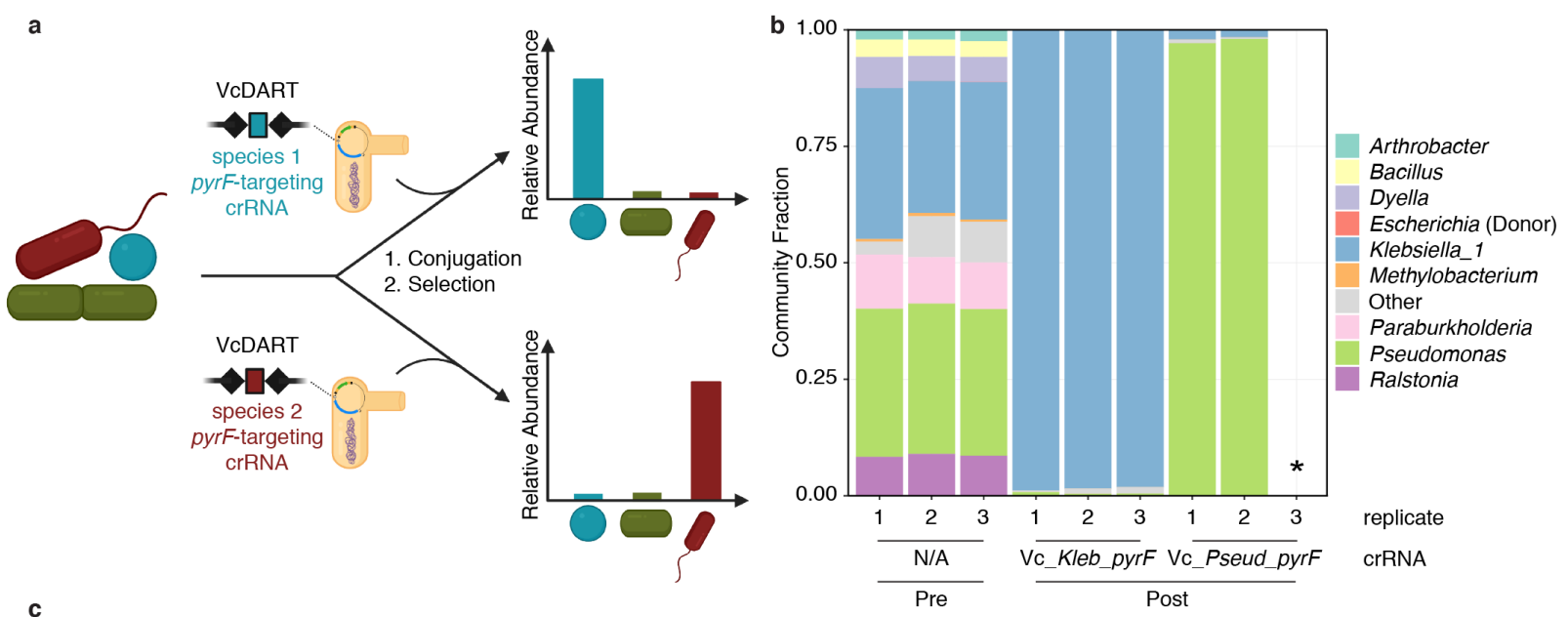

c

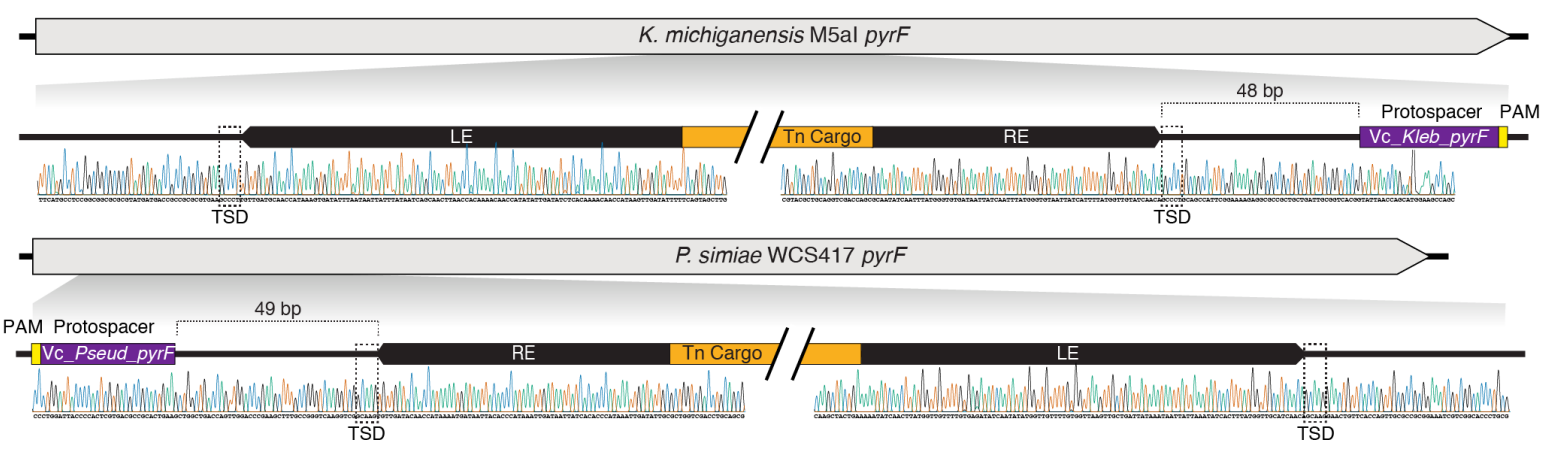

255 Fig. 5 | Targeted editing in the nine-member consortium. a, Conjugative VcDART delivery into a

256 microbial community using species-specific crRNA, followed by selection for transposon cargo, facilitates

257 selective enrichment of targeted organisms. b, Relative abundance of nine-member community 
constituents measured by $16 \mathrm{~S}$ rRNA sequencing before conjugative VcDART delivery and after selection for pyrF-targeted transposition in K. michiganensis or $P$. simiae ( $\mathrm{n}=3$ biological replicates). * indicates no spanning transposon insertion site at targeted pyrF locus in $K$. michiganensis (top) and $P$. simiae (bottom) colonies following VcDART-mediated transposon integration and selection. Target-site duplications (TSD)

263 are indicated with dashed boxes.

\section{Discussion}

266 We have demonstrated organism- and locus-specific genome editing within a microbial

267 community, providing a new approach to microbial genetics and microbiome manipulation for 268 research and applications. ET-Seq revealed the genetic accessibility of organisms growing within 269 microbial communities, including ten microbial species that had not been previously isolated or 270 found to be genetically manipulated. The creation of all-in-one vectors encoding two naturally 271 occurring CRISPR-Cas transposon systems enabled comparison of their targeted genome editing 272 capabilities. These experiments showed that only one of the two systems, which we termed 273 VcDART, enabled precise RNA-programmable microbial genome editing. The ability to conduct

274 targeted genome editing of two bacteria within a nine-member synthetic community and to use

275 the introduced genetic changes as a means of organism isolation demonstrates a new approach

276 to microbiome manipulation. Traditionally, the combined steps of culturing an environmental 277 microbe, determining the ideal means to transform it, and implementing targeted editing could 278 take years or could fail altogether ${ }^{23}$. ET-Seq combined with VcDART compresses the pipeline for 279 establishing genetics in microorganisms to weeks and expands the diversity of organisms that 280 can be targeted beyond those that can be cultivated in isolation.

281 In addition to providing a roadmap for targeted microbial genome editing, ET-Seq can be 282 used to discover and analyze horizontal gene transfer in complex communities. In this study, we 283 observed unexpected community-dependent natural transformation in the nine-member 
community and characterized the horizontal gene transfer events experimentally in the complex microbiome of a thiocyanate-degrading bioreactor. In future experiments, multiple ET-Seq time points could be taken in a community after delivery to measure directly both the portion of the community receiving DNA and the persistence of gene transfer, rather than tracking horizontal gene transfer using bioinformatics ${ }^{24}$ or indirect experimental methods ${ }^{4-9}$. Furthermore, as ET-Seq is applied in increasingly diverse and complex environments, an atlas of editable taxa can be created including optimal delivery approaches. To expand this dataset, we plan to apply ET-Seq

291 to new microbial communities being sampled for metagenomic sequencing, a natural pairing

292 because ET-Seq depends on availability of genome sequences for the component organisms. genome edits. The canonical approach involving antibiotic selection for edited bacteria is

295 infeasible in large complex communities, where many natural resistances exist ${ }^{25,26}$. Even in the 296 nine-member synthetic community used in this study, three antibiotics and counterselection were 297 necessary to achieve strict selection. Improved delivery strategies and alternative positive and counter selection methods should enable more efficient editing. In the gut microbiome, porphyran has been used successfully for selection of a spiked-in organism capable of utilizing this compound $^{27}$. Such approaches for more efficient microbial community editing will enable research

301 to answer fundamental questions as well as allow manipulation of agricultural, industrial, and 302 health-relevant microbiomes. The combination of ET-Seq and DART systems presented here 303 provide the foundation of the new field of in situ microbial genetics.

\section{References}

306 1. Steen, A. D. et al. High proportions of bacteria and archaea across most biomes remain 307 uncultured. ISME J. (2019) doi:10.1038/s41396-019-0484-y.

308 2. Pascual-García, A., Bonhoeffer, S. \& Bell, T. Metabolically cohesive microbial consortia and 
ecosystem functioning. Philos. Trans. R. Soc. Lond. B Biol. Sci. 375, 20190245 (2020).

3. Fux, C. A., Shirtliff, M., Stoodley, P. \& Costerton, J. W. Can laboratory reference strains mirror 'real-world' pathogenesis? Trends Microbiol. 13, 58-63 (2005).

4. Pukall, R., Tschäpe, H. \& Smalla, K. Monitoring the spread of broad host and narrow host range plasmids in soil microcosms. FEMS Microbiol. Ecol. 20, 53-66 (1996).

314 5. De Gelder, L., Vandecasteele, F. P. J., Brown, C. J., Forney, L. J. \& Top, E. M. Plasmid 315 Donor Affects Host Range of Promiscuous IncP-1 $\beta$ Plasmid pB10 in an Activated-Sludge Microbial Community. Appl. Environ. Microbiol. 71, 5309-5317 (2005).

6. Musovic, S., Oregaard, G., Kroer, N. \& Sørensen, S. J. Cultivation-Independent Examination of Horizontal Transfer and Host Range of an IncP-1 Plasmid among GramPositive and Gram-Negative Bacteria Indigenous to the Barley Rhizosphere. Applied and Environmental Microbiology vol. 72 6687-6692 (2006).

7. Musovic, S., Klümper, U., Dechesne, A., Magid, J. \& Smets, B. F. Long-term manure exposure increases soil bacterial community potential for plasmid uptake. Environ. Microbiol. Rep. 6, 125-130 (2014).

8. Klümper, U. et al. Broad host range plasmids can invade an unexpectedly diverse fraction of a soil bacterial community. ISME J. 9, 934-945 (2015).

9. Ronda, C., Chen, S. P., Cabral, V., Yaung, S. J. \& Wang, H. H. Metagenomic engineering of the mammalian gut microbiome in situ. Nat. Methods 16, 167-170 (2019).

10. Farzadfard, F., Gharaei, N., Citorik, R. J. \& Lu, T. K. Efficient Retroelement-Mediated DNA Writing in Bacteria. bioRxiv (2020).

11. Hsu, B. B., Way, J. C. \& Silver, P. A. Stable Neutralization of a Virulence Factor in Bacteria Using Temperate Phage in the Mammalian Gut. mSystems 5, (2020). in situ Microbiome Engineering. Trends Genet. 32, 189-200 (2016).

334 13. Wang, X. et al. Across Genus Plasmid Transformation Between Bacillus subtilis and 
Escherichia coli and the Effect of Escherichia coli on the Transforming Ability of Free

Plasmid DNA. Current Microbiology vol. 54 450-456 (2007).

14. Borgeaud, S., Metzger, L. C., Scrignari, T. \& Blokesch, M. The type VI secretion system of Vibrio cholerae fosters horizontal gene transfer. Science 347, 63-67 (2015).

15. Kantor, R. S. et al. Genome-Resolved Meta-Omics Ties Microbial Dynamics to Process Performance in Biotechnology for Thiocyanate Degradation. Environ. Sci. Technol. 51, 2944-2953 (2017).

16. Huddy, R. J. et al. Thiocyanate and organic carbon inputs drive convergent selection for specific autotrophic Afipia and Thiobacillus strains within complex microbiomes. bioRxiv 2020.04.29.067207 (2020) doi:10.1101/2020.04.29.067207.

17. Castelle, C. J. et al. Biosynthetic capacity, metabolic variety and unusual biology in the CPR and DPANN radiations. Nat. Rev. Microbiol. 16, 629-645 (2018).

18. Strecker, J. et al. RNA-guided DNA insertion with CRISPR-associated transposases.

349 19. Klompe, S. E., Vo, P. L. H., Halpin-Healy, T. S. \& Sternberg, S. H. Transposon-encoded CRISPR-Cas systems direct RNA-guided DNA integration. Nature 571, 219-225 (2019).

351 20. Petassi, M. T., Hsieh, S.-C. \& Peters, J. E. Guide RNA categorization enables target site choice in Tn7-CRISPR-Cas transposons. bioRxiv 2020.07.02.184150 (2020) doi:10.1101/2020.07.02.184150.

21. Rice, P. A., Craig, N. L. \& Dyda, F. Comment on 'RNA-guided DNA insertion with CRISPRassociated transposases'. Science 368, (2020).

22. Strecker, J., Ladha, A., Makarova, K. S., Koonin, E. V. \& Zhang, F. Response to Comment on 'RNA-guided DNA insertion with CRISPR-associated transposases'. Science $\mathbf{3 6 8 ,}$

359 23. Laurenceau, R. et al. Toward a genetic system in the marine cyanobacterium 360 Prochlorococcus. Access Microbiology 6, 23 (2020). 
361 24. Soucy, S. M., Huang, J. \& Gogarten, J. P. Horizontal gene transfer: building the web of life. Nat. Rev. Genet. 16, 472-482 (2015).

363 25. Hu, Y. et al. Metagenome-wide analysis of antibiotic resistance genes in a large cohort of 364 human gut microbiota. Nat. Commun. 4, 2151 (2013).

365 26. Forsberg, K. J. et al. Bacterial phylogeny structures soil resistomes across habitats. Nature $366 \quad 509,612-616(2014)$.

367 27. Shepherd, E. S., DeLoache, W. C., Pruss, K. M., Whitaker, W. R. \& Sonnenburg, J. L. An 368 exclusive metabolic niche enables strain engraftment in the gut microbiota. Nature 557, $369 \quad$ 434-438 (2018). 


\section{Methods}

\section{Plasmid construction and barcoding}

373 For ET-Seq measurement of genetic tractability in community members, DNA encoding a non-

374 targeted mariner transposon was delivered. The mariner transposon integrates into "TA" 375 sequences in recipient cells. For delivery of the mariner transposon, we made use of the 376 previously created pHLL250 vector, which contains an RP4 origin of transfer (oriT), AmpR, 377 conditional ( pir $^{+}$-dependent) R6K origin, and an Asel restriction site to facilitate depletion of vector 378 from DNA samples in ET-Seq library preparations ${ }^{1}$. Unique to each transposon on this vector is 379 a random $20 \mathrm{bp}$ barcode sequence to aid in the discrimination of unique insertion events from 380 duplications of the same insertion due to cell division or PCR.

DART vectors were designed to encode all components required for delivery and editing

382 (Supplementary Table 2 and Extended Data Fig. 4). VcCasTn genes, crRNA, and Tn were 383 synthesized as gBlocks (IDT). pHelper_ShCAST_sgRNA was a gift from Feng Zhang (Addgene 384 plasmid \#127921; http://n2t.net/addgene:127921; RRID:Addgene_127921) and was used to 385 clone ShCasTn genes and sgRNA. pDonor_ShCAST_kanR was a gift from Feng Zhang 386 (Addgene plasmid \# 127924 ; http://n2t.net/addgene:127924 ; RRID:Addgene_127924) and was 387 used to clone the ShCasTn transposon. tns genes, cas genes, and crRNA/sgRNA were 388 consolidated into a single operon (with various promoters and transcriptional configurations) on 389 the same vector as the cognate transposon. The left end of the cognate Tn was encoded 390 downstream of the crRNA/sgRNA, followed by Tn cargo, barcode, and Tn right end. DART Tn LE 391 and RE were designed to include the minimal sequence that both included all putative TnsB 392 binding sites and was previously shown to be functional ${ }^{2,3}$. Specifically, VcDART LE (108 bp) and 393 RE (71 bp) each encompass three 20 bp putative TnsB binding sites, spanning from the edge of 394 the $8 \mathrm{bp}$ terminal ends to the edge of the third putative TnsB binding site ${ }^{2}$. ShDART LE (113 bp) 395 spans the boundaries of the long terminal repeat and both additional putative TnsB binding sites, 
while the RE (211 bp) encompasses the long terminal repeat and all four additional putative TnsB binding sites ${ }^{3}$.

Vectors were cloned using Bbsl (NEB) Golden Gate assembly of part plasmids, each encoding different regions of the final plasmid. Of note, the backbone encodes RP4 oriT, AmpR, conditional R6K origin, and an AsiSI+Sbfl double digestion site for vector depletion during ET-

401 Seq library preparations. A 2xBsal spacer placeholder enabled spacer cloning with Bsal (NEB)

402 Golden Gate. A 2xBsmBI barcode placeholder was encoded immediately inside the Tn right end 403 and was used for barcoding as described below. Part plasmids were propagated in E. coli Mach1-

404 T1R (QB3 Macro Lab). Golden Gate reactions for all-in-one vector assembly were purified with 405 DNA Clean \& Concentrator-5 (Zymo Research) and electroporated into E. coli EC100D-pir+ 406 (Lucigen).

DART vectors were barcoded by BsmBI (NEB) Golden Gate insertion of random barcode 408 PCR product into the $2 x B s m B I$ barcode placeholder using a previously reported method ${ }^{4}$ with 409 slight modifications. A 56-nt ssDNA oligonucleotide encoding a central tract of 20 degenerate 410 nucleotides (oBFC1397) was amplified with BsmBI-encoding primers oBFC1398 and oBFC1399 411 using Q5 High-Fidelity 2 X Master Mix (NEB) in a six-cycle PCR $\left(98^{\circ} \mathrm{C}\right.$ for $1 \mathrm{~min}$; six cycles of $98^{\circ} \mathrm{C}$ 412 for $10 \mathrm{~s}, 58^{\circ} \mathrm{C}$ for $30 \mathrm{~s}$, and $72^{\circ} \mathrm{C}$ for $60 \mathrm{~s}$; and $72^{\circ} \mathrm{C}$ for $\left.5 \mathrm{~min}\right)$. Barcoding Golden Gate reactions 413 were purified with DNA Clean \& Concentrator-5. To remove residual non-barcoded vector, 414 reactions were digested with $15 \cup \mathrm{BsmBI}$ at $55^{\circ} \mathrm{C}$ for at least $4 \mathrm{hr}$, heat inactivated at $80^{\circ} \mathrm{C}$ for 20 $415 \mathrm{~min}$, treated with $10 \mathrm{U}$ Plasmid-Safe ATP-Dependent DNase (Lucigen) exonuclease at $37^{\circ} \mathrm{C}$ for $4161 \mathrm{hr}$, heat inactivated at $70^{\circ} \mathrm{C}$ for $30 \mathrm{~min}$, and purified with DNA Clean \& Concentrator-5. Randomly barcoded conjugative vectors were electroporated into E. coli EC100D-pirt, 418 followed $1 \mathrm{hr}$ recovery in $1 \mathrm{~mL}$ pre-warmed SOC (NEB) at $37^{\circ} \mathrm{C} 250 \mathrm{rpm}$, serial dilution and spot 419 plating on LB agar plus $100 \mu \mathrm{g} \mathrm{mL}^{-1}$ carbenicillin to estimate library diversity, and plating the full 420 transformation across 5 LB agar plates containing carbenicillin (and other appropriate antibiotics 421 when Tn cargo contained other resistance cassettes). To prepare barcoded conjugative vector 
422 plasmid stock, all 5 agar plates were scraped into a single pool and midiprepped (Zymo

423 Research). All conjugations were performed using the diaminopimelic acid (DAP) auxotrophic

424 RP4 conjugal donor E. coli strain WM3064. Donor strains were prepared by electroporation with

$425200 \mathrm{ng}$ barcoded vectors, followed by recovery in SOC plus DAP at $37^{\circ} \mathrm{C}$ and $250 \mathrm{rpm}$ and

426 inoculation of the entire recovery culture into $15 \mathrm{~mL} \mathrm{LB}$ containing DAP and carbenicillin in $50 \mathrm{~mL}$

427 conical tubes, followed by overnight cultivation at $37^{\circ} \mathrm{C}$ and $250 \mathrm{rpm}$. Donor serial dilutions were

428 spot plated on LB agar plus carbenicillin to estimate final barcode diversity.

\section{Guide RNA design}

431 In all experiments, VcCasTn gRNAs used 32 nt spacers and a 5'-CC Type IF PAM, while

432 ShCasTn gRNAs used 23 nt spacers and a 5'-GTT Cas12k PAM. All gRNAs were designed to 433 bind in the first half of the target CDS to ensure functional knockout by transposon insertion 434 (Supplementary Table 3). Off-target potential was assessed using BLASTn (-dust no -word_size 4354 ) of spacers against a local BLAST database created from all genomes present in an experiment, 436 and spacers were discarded if off-target hits with E-value $<15$ were identified. gRNAs with less 437 seed region complementarity to off-targets were prioritized. Non-targeting gRNAs were designed 438 by scrambling the spacer until no significant matches were found.

\section{Delivery methods}

441 For natural transformation and electroporation, a culture of the community or isolate to be

442 transformed was subcultured at $\mathrm{OD}_{600}=0.2$ and grown to $\mathrm{OD}_{600}=0.5$. In the case of the 443 thiocyanate-degrading bioreactor in the absence of accurate OD measurements the culture was 444 outgrown for two hours. For natural transformation $200 \mathrm{ng}$ of vector harboring the mariner 445 transposon $\left(\mathrm{pHLL}_{250^{1}}\right.$ ) for non-targeted insertion, or water for the negative control were added 446 to $4 \mathrm{~mL}$ of $\mathrm{OD}_{600}=0.5$ outgrowth. Cultures were incubated for 3 hours shaking at $250 \mathrm{rpm}$ at 
447 temperature appropriate for the isolate or community before being moved to the appropriate 448 downstream analysis.

449 For electroporation, $20 \mathrm{~mL}$ of the community or isolate at $\mathrm{OD}_{600}=0.5$ was put on ice, 450 centrifuged at $4,000 \mathrm{~g}$ at $4^{\circ} \mathrm{C}$ for 10 minutes, and washed four times with $10 \mathrm{~mL}$ sterile ice-cold 451 Milli-Q $\mathrm{H}_{2} \mathrm{O}$. After a final centrifugation the pellet was resuspended in $100 \mu \mathrm{L}$ of $2 \mathrm{ng} / \mu \mathrm{L}$ vector 452 (pHLL250 or VcDART), or $100 \mu \mathrm{L}$ of water as a negative control. This solution was then pipetted 453 into a $0.2 \mathrm{~cm}$ gap ice-cold cuvette and electroporated at $3 \mathrm{kV}, 200 \Omega$, and $25 \mu \mathrm{F}$. The cells were

454 immediately recovered into $10 \mathrm{~mL}$ of the community's or isolate's preferred medium and incubated 455 shaking for 3 hours before being moved to the appropriate downstream analysis.

456 E. coli strain WM3064 containing the mariner transposon (pHLL250) for non-targeted 457 editing, or the VcDART for targeted editing was cultured overnight in LB supplemented with 458 carbenicillin $(100 \mu \mathrm{g} / \mathrm{mL})$ and DAP $(60 \mu \mathrm{g} / \mathrm{mL})$ at $37^{\circ} \mathrm{C}$. Before conjugation the donor strain was 459 washed twice in LB (centrifugation at $4,000 \mathrm{~g}$ for 10 minutes) to remove antibiotics. Then, 1 $460 \mathrm{OD}_{600}{ }^{*} \mathrm{~mL}$ of the donor was added to $1 \mathrm{OD}_{600}{ }^{*} \mathrm{~mL}$ of the recipient community or isolate and the 461 mixture was plated on a $0.45 \mu \mathrm{m}$ mixed cellulose ester membrane (Millipore) topping a plate of 462 the recipient's preferred media without DAP. In the case of the thiocyanate-degrading bioreactor, $463 \sim 2 \mathrm{OD}_{600}{ }^{*} \mathrm{~mL}$ of the donor was added to $2 \mathrm{OD}_{600^{*}} \mathrm{~mL}$ of the recipient community to ensure 464 sufficient material despite the community's slow growth. Plates were incubated at the ideal 465 temperature for the recipient community or isolate for 12 hours before the growth was scraped off 466 the filter into the media of the recipient community or isolate for downstream analysis.

\section{$468 \quad$ ET-Seq library preparation}

469 The insertion junction sequencing library prep strategy for ET-Seq can be used (modification may 470 be necessary) in any circumstance where high efficiency mapping of inserted DNA to a host loci 471 is desired. For our purposes, DNA of the edited community or isolate was first extracted using 
472 the DNeasy PowerSoil Kit (QIAGEN). In the case of the nine-member community, 500 ng of DNA

473 was used for both insertion junction sequencing and metagenomic library prep. For the SCN

474 community, which had lower yields of DNA, $100 \mathrm{ng}$ were used. As an internal standard, DNA

475 from a previously constructed mutant library of Bacteroides thetaiotaomicron VPI-5482 ${ }^{5}$, a

476 species not present in the nine-member community or the thiocyanate-degrading bioreactor, was

477 spiked into the community DNA at a ratio of $1 / 500$ by mass. The $B$. thetaiotaomicron library had

478 undergone antibiotic selection for its transposon insertions and was thus assumed to represent

$479100 \%$ transformation efficiency (i.e. every genome contained at least one mariner transposon 480 insertion).

481 For metagenomic sequencing, library prep was conducted by the standard $\geq 100 \mathrm{ng}$ 482 protocol from the NEBNext Ultra II FS DNA Library Prep Kit for Illumina (NEB). For insertion 483 junction sequencing, the same protocol was used with a number of modifications enumerated 484 here (Extended Data Fig. 1). This insertion junction sequencing protocol has also been tested 485 successfully with the $\leq 100 \mathrm{ng}$ protocol of the NEBNext Ultra II FS DNA Library Prep Kit (NEB) 486 and the KAPA HyperPlus Kit (Roche). For fragmentation an 8 minute incubation was used. A 487 custom splinklerette adaptor was used during adaptor ligation to decrease non-specific 488 amplification (Supplementary Table 4$)^{6,7}$. For size selection $0.15 X$ (by volume) SPRIselect 489 (Beckman Coulter, Cat \# B23318) or NEBNext Sample Purification Beads (NEB) were used for 490 the first bead selection and $0.15 X$ (by volume) were added for the second. From this selection, 491 the DNA was eluted in $44 \mu \mathrm{L}$ (instead of the suggested $15 \mu \mathrm{L}$ ) where it undergoes digestion before 492 enrichment to cleave intact transposon delivery vector. All bead elutions were performed with 493 Sigma Nuclease-Free water. pHLL250 underwent Asel digestion, while DART vectors underwent 494 double digestion by AsiSI and Sbfl-HF (NEB) (Supplementary Table 2). The DNA then underwent 495 a sample purification using $1 \mathrm{X}$ AMPure XP beads (Beckman Coulter) to prepare it for PCR 496 enrichment. 
In PCR enrichment, the transposon junction was amplified by nested PCR. The PCRs

498 followed the NEBNext Ultra II FS DNA Library Prep Kit for Illumina (NEB) PCR protocol, however

499 in the first PCR the primers were custom to the transposon and the adaptor and the PCR was run

500 for 25 cycles (Supplementary Table 4). The enrichment then underwent sample purification with

501 a $0.7 X$ size selection using SPRIselect or NEBNextSample Purification Beads from which $15 \mu \mathrm{L}$

502 were eluted for the second PCR. This second PCR used custom unique dual indexing primers

503 specific to nested regions of the insertion and adaptor and 6 cycles are used (Supplementary

504 Table 4). Then another $0.7 x$ size selection was conducted and the final library was eluted in 30

$505 \mu \mathrm{L}$. Samples for metagenomic sequencing and insertion junction sequencing were then quality

506 controlled and multiplexed using 1X HS dsDNA Qubit (Thermo Fisher) for total sample

507 quantification, Bioanalyzer DNA 12000 chip (Agilent) for sizing, and qPCR (KAPA) for

508 quantification of sequenceable fragments. Samples were sequenced on the iSeq100 or

509 HiSeq4000 platforms.

511 Genome sequencing, assembly, taxonomic classification, and database construction

512 For a full list of genome sequences used as read mapping references in this study see

513 Supplementary Table 1. Assembly and annotation of genomes used as references for the SCN

514 bioreactor experiment is described in Huddy et al. ${ }^{8}$. As the SCN bioreactor has been subjected to

515 numerous genome-resolved metagenomic studies ${ }^{8,9}$ we endeavored to create a non-redundant

516 database that contained all genomes previously observed in this reactor system. A set of 556

517 genomes assembled from this system were de-replicated at the species level using dRep v2.5.3 ${ }^{10}$

518 with an average nucleotide identity (ANI) threshold of $95 \%$ and a minimum completeness of $60 \%$

519 as estimated by checkM v1.1.2 ${ }^{11}$. A single genome representing each species level group was

520 chosen by dRep based on optimizing genome size, fragmentation, estimated completeness, and

521 estimated contamination resulting in 265 representative genomes. Genomes were taxonomically

522 classified using GTDB-Tk ${ }^{12}$ with default options. Additionally, to display the taxonomic diversity of 
523 transformed organisms (Fig. 3b), a phylogenetic tree was constructed using ribosomal protein S3

524 (rpS3). Briefly, a custom Hidden Markov Model (HMM) was used to identify rpS3 sequences ${ }^{13}$ in

525 the 265 representative genomes, and 262 rpS3 sequences were successfully identified.

526 Sequences were aligned using muscle ${ }^{14}$, and a maximum likelihood phylogenetic tree was

527 constructed using IQ-TREE ${ }^{15}$. Phylogenetic trees were pruned and annotated using iTol v5

528 (https://itol.embl.de/). To determine if an organism we identified as receiving exogenous DNA was

529 ever previously isolated we used the ANI relative to the closest reference genome in the GTDB-

530 Tk output. If one of our genomes had an $\mathrm{ANI} \geq 95 \%$ relative to a known reference, and this

531 reference genome was generated from an isolated bacterium, our target organism was

532 considered to be previously isolated (Supplementary Table 1).

For 9-member community genomes assembled as part of this study, cultures were grown

534 on R2A medium for 24 hours at $30^{\circ} \mathrm{C}$ and genomic DNA was extracted with the DNeasy Blood

535 and Tissue DNA Kit (Qiagen) with pre-treatment for Gram-positive bacteria. Genomic DNA was

536 sheared mechanically with the Covaris S220 and processed with the NEBNext DNA Library Prep

537 Master Mix Set for Illumina (NEB) before submitting for sequencing on an Illumina MiSeq platform

538 generating paired end 150 bp reads. Raw sequencing reads were processed to remove Illumina

539 adapter and phiX sequence using BBduk with default parameters, and quality trimmed at 3' ends

540 with Sickle using default parameters (https://github.com/najoshi/sickle). Assemblies were

541 conducted using IDBA-UD v1.1.1 $1^{16}$ with the following parameters: -pre_correction -mink 30 -

542 maxk 140 -step 10. Following assembly, contigs smaller than $1 \mathrm{kbp}$ were removed and open

543 reading frames (ORFs) were then predicted on all contigs using Prodigal v2.6.3 $3^{17}$. $16 \mathrm{~S}$ ribosomal

544 rRNA genes were predicted using the 16SfromHMM.py script from the ctbBio python package

545 using default parameters (https://github.com/christophertbrown/bioscripts). Transfer RNAs were

546 predicted using tRNAscan-SE ${ }^{18}$. The full metagenome samples and their annotations were then

547 uploaded into our in-house analysis platform, ggKbase, where genomes were manually curated 
548 via the removal of contaminating contigs based on aberrant phylogenetic signatures

549 (https://ggkbase.berkeley.edu).

550 For each ET-Seq experiment a genomic database is constructed using the ETdb 551 component of the ETsuite software package. Each database contains the nucleotide sequences

552 of the expected organisms in a sample, any vectors used, any conjugal donor, and the spike in

553 control organism. Briefly, all genomic sequences are formatted into a bowtie2 index to allow read

554 mapping, a tabular correspondence table between all scaffold names and their associated

555 genome is constructed (scaff2bin.txt), and a table (genome_info.txt) of standard genomic

556 statistics is calculated including genome size, GC content, and number of scaffolds. Following

557 database construction, a label is manually added to each entry in the genome info table to indicate

558 if the entry represents a target organism, a vector, or a spike in control organism. All data are

559 propagated into a single folder that can be used by the ETmapper software for downstream

560 mapping and analysis.

561

562 Identification and quantification of insertion junctions and barcodes

563 To identify and map transposon insertion junctions and their associated barcodes in a mixed

564 population of microbial cells, reads (150 bp X 2) generated from PCR amplicons of putative

565 transposon insertion junctions are first processed using the ETmapper component of the ETsuite

566 software package implemented in $\mathrm{R}$ with the following steps: First reads are quality trimmed at

567 the 3 ' end to remove low quality bases (Phred score $\leq 20$ ) and sequencing adapters using

568 Cutadapt v2.10 19 . Cutadapt is then used to identify and remove provided transposon model

569 sequences from the 5' end of forward reads, requiring a match to $95 \%$ of the shortest transposon

570 sequence in a provided set and allowing a $2 \%$ error rate. Read pairs where no transposon model

571 sequence is identified in the forward read are discarded. All identified and trimmed transposon

572 models are paired with their respective reads, stored, and barcodes are identified in these

573 sequences by searching for a known primer binding site sequence flanking the 5 ' end of the 
574 barcode (5'-CTATAGGGGATAGATGTCCACGAGGTCTCT-3') allowing for 1 mismatch.

575 Subsequently, the 20 bp region following the known primer binding site is extracted as the barcode

576 sequence and associated with its respective read. The 3' end of the paired reverse reads are then

577 trimmed to remove any transposon model sequence using Cutadapt, and only read pairs where

578 one mate is at least $\geq 40 \mathrm{bp}$ following all trimming are retained for downstream mapping and

579 analysis. The fully trimmed paired end reads now consisting of only genomic sequence following

580 the transposon insertion site are mapped to the ETdb database used in a given experiment using

581 bowtie2 with default options ${ }^{20}$. Mapped read files are converted into a hit table indicating the

582 mapped genome, scaffold, genomic coordinates, mapQ score, and number of alignment

583 mismatches for each read in a pair using a custom Python script, bam_pe_stats.py, provided with

584 ETsuite. This table is then merged with read-barcode assignments to generate a final hit table

585 with the mapping information about each read pair, the transposon model identified, and the

586 associated barcode found for that read pair. Finally mapped read pairs are only retained for

587 downstream quantification if both reads map to the same genome, at least one mapped read in a

588 pair has a mapQ score $\geq 20$, and a barcode was successfully identified and associated with the

589 read pair.

To quantify the number of unique barcodes and their associated reads mapping to

591 organisms in each sample of an experimental run, the filtered hit tables were processed using the

592 ETstats component of the ET-Seq software package with the following steps: Initially, all barcodes

593 identified across all samples in an experiment are aggregated and clustered using Bartender ${ }^{21}$

594 with the following supplied options: $-1 \quad 4$-s 1 -d 3 . Barcode clusters and their associated

595 barcodes/reads were only retained if all of the following criteria were true: (1) $\geq 75 \%$ of the reads

596 in a cluster mapped to one genome (the majority genome), (2) $\geq 75 \%$ of the reads in a cluster

597 were associated with the same transposon model (the majority model), and (3) the barcode

598 cluster had at least 2 reads. Subsequently, when quantifying reads and barcodes in each sample

599 of an experiment, the genome a read was mapped to and the transposon model it was associated 
600 with had to agree with the majority assignments for the barcode cluster assigned to that read's

601 barcode to be counted. Finally, we were aware that Illumina patterned flow cell related index

602 swapping would result in reads from a barcode cluster being misassigned across samples, even

603 when using unique dual indexing ${ }^{22}$. We could not simply limit barcode clusters to be associated

604 with only one sample, as our spike in control organisms contain the same pool of barcodes and

605 are added to every sample. Thus we estimated an empirical index swap rate across each

606 experiment and required that the number of reads $(X)$ for a barcode to be positively identified in

607 a sample be always $\geq 2$ and $\geq$ the binomial mean of observed read counts expected in any sample

608 for a barcode cluster with $(\mathrm{R})$ reads across $(\mathrm{N})$ samples based on the estimated swap rate $(\mathrm{S})+$ 6092 standard deviations (Eqn. 1)

610

611 Eqn. 1: $X \geq\left(R \times\left(\frac{S}{N}\right)\right)+2 \times \sqrt{R \times(1-S) \times S} \& X \geq 2$

613 The index swap rate for an experiment was empirically estimated from barcode clusters assigned

614 only to target organisms based on the assumption that it would be highly unlikely for a barcode

615 cluster to have truly originated from independent integration events into the same organism in

616 more than one sample. Thus we assumed that for each barcode cluster associated with target

617 organisms, the majority of reads originated from the true sample and reads assigned to other

618 samples represented swaps. This is opposed to barcode clusters associated with our spike-in

619 organism, conjugal donor organism, or vectors which contain the same pool of barcodes directly

620 added to multiple samples. To identify swapped read counts we first quantify the total count of all

621 reads assigned to the majority genome across barcode clusters but that are not associated with

622 the majority sample of that cluster $(E)$. Then we quantify the total count of reads associated with

623 the majority genome and associated with the majority sample across all clusters (C). Then 
624 experiment wide swap rate was estimated by dividing the total number of reads not associated

625 with majority samples by the total number of reads (Eqn. 2)

626

627 Eqn. 2: $S=\frac{E}{(E+C)}$

629 Following filtering, a hit table is returned that indicates for each genome in each sample, the

630 number of unique barcode clusters that were recovered, and the total number of reads associated

631 with these barcodes. As a final check for false positives during ET-Seq development we included

632 an organism genome as an ETsuite mapping target, Sinorhizobium meliloti, which was not

633 physically included in our 9-member synthetic or thiocyanate-degrading communities. We did not

634 detect any barcodes or reads associated with this genome.

636 Metagenomic data processing and coverage calculation.

637 Each ET-Seq sample is split and in parallel undergoes shotgun metagenomic sequencing to 638 determine the relative quantities of organisms present in the sample at the time of sampling. Raw 639 read files from metagenomic data are also processed using the ETmapper component of the

640 ETsuite software package with the following steps: First reads are quality trimmed at the 3' end

641 to remove low quality bases (Phred score $\leq 20$ ) and sequencing adapters using Cutadapt v2.10 ${ }^{19}$.

642 Read pairs where at least one mate is not $\geq 40 \mathrm{bp}$ in length are discarded. Trimmed read pairs

643 are mapped to the ETdb database used in a given experiment using bowtie $2^{20}$ with default

644 parameters. Mappings are filtered to require a minimum identity $\geq 95 \%$ and minimum mapQ score

$645 \geq 20$, and coverage is calculated using a custom script, calc_cov.py, included with the ETsuite 646 software. 
647 Metagenomic sequencing for one biological replicate of the thiocyanate-degrading bioreactor

648 community (Conjugation - Control Sample - Replicate 3) failed. Metagenomic coverage values for

649 this replicate were generated by averaging the values from the other two biological replicates.

\section{ET-Seq normalization and calculation of insertion efficiency.}

652 To account for differences in sequencing depth, transposon junction PCR template amount, and 653 relative abundance of microbes in a community the data generated from both ET-Seq and 654 shotgun metagenomics were each normalized independently to values from the spike in control 655 organism, B. thetaiotaomicron, and then ET-Seq data is subsequently normalized by 656 metagenomic abundance as follows: Initially read count tables from ET-Seq and metagenomics 657 are filtered to remove any ET-Seq read count associated with $<2$ barcodes and any metagenomic 658 read count $<10$ reads. Next a size factor for each sample is calculated based on the geometric 659 mean of $B$. thetaiotaomicron reads for ET-Seq samples and $B$. thetaiotaomicron coverage for 660 metagenomics samples. ET-Seq read counts and metagenomic coverage values are then divided

661 by their respective sample size factors to create normalized values. Normalized ET-Seq read 662 counts are then divided by their paired normalized metagenomic coverage values to generate ET663 Seq read counts that are fully normalized to both ET-Seq sequencing depth and metagenomic 664 coverage. Finally fully normalized ET-Seq read counts for target organisms are divided by the 665 fully normalized ET-Seq read count of $B$. thetaiotaomicron from an experiment (a constant that 666 represents the number of reads that would be obtained from an organism with $100 \%$ of its 667 chromosomes carrying insertions). The resulting values for each target organism in a sample 668 represent an estimate of the fraction of that organism's population that received insertions 669 (Insertion Efficiency). Additionally, we multiply a target organism's insertion efficiency by the 670 fractional relative abundance of that organism in a sample, based on metagenomic data, to 671 estimate the fraction of an entire sample population that is made up of cells of a given species 672 that received insertions (Insertion-Receiving Fraction in Total Community). 
674 ET-Seq validation and establishing limits of detection and quantification.

675 To validate ET-Seq and establish both a limit of detection (LOD) and limit of quantification (LOQ)

676 for the assay, a library of $K$. michiganensis transposon mutants was constructed by antibiotic

677 selection following conjugation with pHLL250 (as described above), and this library was added to

678 untransformed samples of the combined nine-member community to create a transformed cell

679 concentration gradient. Technical triplicate samples were created where $1 \%, 0.1 \%, 0.01 \%$,

$680 \quad 0.001 \%$ and $0 \%$ of the total $K$. michiganensis cells (by $\mathrm{OD}_{600}$ ) in the mixture were those derived

681 from the transformed library. All samples $(n=15)$ were subjected to ET-Seq (as described above),

682 and pooled samples across all concentrations for each technical triplicate $(n=3 ; 5$ concentrations $)$

683 were analyzed for community composition using shotgun metagenomics (as described above).

684 ET-Seq insertion efficiencies and insertion-receiving fraction in total community values were

685 averaged across technical replicates. Additionally, to derive the fraction of transformed $K$.

686 michiganensis cells that made up the total community (not just the $K$. michiganensis sub-

687 population), the known fraction of $K$. michiganensis cells that were transformed in a sample was

688 multiplied by the measured relative abundance of $K$. michiganensis in a given technical replicate,

689 and these values were averaged across technical replicates.

To derive the LOD and LOQ for ET-Seq a linear regression was performed using the Im

691 function in the base package of $\mathrm{R}^{23}$ using the known fraction of transformed $K$. michiganensis

692 cells that made up the total community as the independent variable and the ET-Seq estimated

693 per community insertion efficiency as the dependent variable. The sample where transformed $K$.

694 michiganensis made up $0 \%$ of the community was not included in the regression analysis, but

695 was reserved to demonstrate zero response with no transformed cells present. LOD was

696 calculated as $3.3^{*}$ standard error of the regression / slope. The LOQ was calculated as 10 *

697 standard error of the regression / slope.

698 


\section{Identification of positive transformations and statistical analysis}

700 For all ET-Seq experiments conducted we initially determined if any ET-Seq estimated per

701 community insertion efficiency was larger than the LOD. Values larger than the LOD constituted

702 a positive detection. For comparative statistical analysis conducted to compare insertion

703 efficiencies between transformation methods (Fig. 2b) only values that had a corresponding

704 insertion-receiving fraction in total community > LOQ were used. Statistical testing was conducted

705 using Analysis of Variance (ANOVA) implemented in the aov function in $\mathrm{R}^{23}$. Post-hoc testing was

706 conducted using the TukeyHSD function in R. Traditional 95\% confidence intervals were

707 calculated using the groupwiseMean function of the rcompanion package in $\mathrm{R}$.

708

709 Multiple delivery experiments in communities.

710 To test multiple delivery methods on the nine-member community, all members were grown at $71130^{\circ} \mathrm{C}$ with Bacillus sp. AnTP16 and Methylobacterium sp. UNC378MF in R2A liquid media while

712 all other members were inoculated in LB. Equal amounts of community members were then

713 combined by $\mathrm{OD}_{600}$. This consortium then underwent transformation (of pHLL250), conjugation

714 (pHLL250 in WM3064), and electroporation of the pHLL250 vector (described in Delivery Methods

715 section). After delivery the community was spun down at 5,000g for 10 minutes, washed once

716 with LB and then spun down and frozen at $-80^{\circ} \mathrm{C}$ until genomic DNA extraction.

717 The thiocyanate-degrading microbial community was sampled for delivery testing from biofilm on

718 a four liter continuously stirred tank reactor that had been maintained at steady state for over a

719 year. The reactor is operated with a two day hydraulic residence time, sparged with laboratory air

720 at $0.9 \mathrm{~L} / \mathrm{min}$, and fed with a mixture of molasses $(0.15 \% \mathrm{w} / \mathrm{v})$, thiocyanate $(250 \mathrm{ppm})$, and $\mathrm{KOH}$

721 to maintain $\mathrm{pH}$ 7. OD measurements were not feasible on the biofilm so we used its wet mass to

722 approximate equivalent OD and thus cell numbers to those used for the nine-member community.

723 This community underwent the same transformation, electroporation, and conjugation delivery

724 approaches as the nine-member community, however in all steps requiring media, LB was 
725 replaced with molasses media (no thiocyanate). After delivery the community was spun down at

$7265,000 \mathrm{~g}$ for 10 minutes, washed once with molasses media and then spun down and frozen at -

$72780^{\circ} \mathrm{C}$ until genomic DNA extraction.

728

729 Benchmarking DART systems in E. coli.

730 We first constructed several DART systems to identify variants capable of efficient transposition

731 by conjugative delivery to $E$. coli. We performed parallel conjugation of each DART vector variant

732 containing $\mathrm{Gm}^{\mathrm{R}}$ Tn cargo (2.1 kbp) and either a non-targeting gRNA or one of two lacZ-targeting

733 gRNAs for each system. For VcDART, variation of the promoter controlling the expression of

734 VcCasTn components did not significantly impact transposition efficiency (Extended Data Fig. 4c-

735 d). Similarly for ShDART, expression of the sgRNA in three distinct transcriptional configurations

736 did not significantly impact transposition efficiency (Extended Data Fig. 4e-f). Since promoter and

737 transcriptional configuration variation had insignificant effects on transposition efficiency--and to

738 remove the requirement for promoter induction and reliance on T7 RNA polymerase--we

739 performed target specificity benchmarking of VcDART and ShDART using the same constitutive

$740 \mathrm{P}_{\text {lac }}$ promoter. In this experiment, ShDART Cas and Tns genes and sgRNA were encoded in the

741 original transcriptional configuration and under control of the same promoter in which ShCasTn

742 was first characterized by Strecker et al. ${ }^{3}$.

743 The lacZ-targeting gRNAs were designed to target the lacZ a-peptide present in the

744 conjugation recipient strain $E$. coli BL21(DE3) but absent in the lacZ $\Delta M 15$ strains used as cloning

745 host (E. coli EC100D-pir+) or conjugation donor (E. coli WM3064), preventing transposition until

746 delivery into the recipient cell (Extended Data Fig. 4a). Donor WM3064 strains were transformed

747 and cultivated as described above, and recipient BL21(DE3) was inoculated from glycerol stock

748 into $100 \mathrm{~mL} \mathrm{LB}$ in a $250 \mathrm{~mL}$ baffled shake flask at $37^{\circ} \mathrm{C} 250 \mathrm{rpm}$. Conjugations were performed

749 as described above using LB medium and $37^{\circ} \mathrm{C}$ incubation for every step, except that $0.1 \mathrm{mM}$

750 IPTG was added to VcDART conjugation plates in Extended Data Fig. 4d to induce transcription 
751 from $\mathrm{P}_{\mathrm{T} 7 \text {-lac }}$ and T7 RNA polymerase expression in E. coli BL21(DE3). Transposition efficiencies

752 were calculated as the percentage of colonies resistant to $10 \mu \mathrm{g} \mathrm{mL}^{-1}$ gentamycin relative to viable

753 colonies in absence of gentamycin.

754 On/off-target analysis was performed for one lacZ-targeting guide for each DART system

755 by outgrowth under selection followed by genomic DNA extraction and ET-Seq. Specifically,

756 approximately 10,000 transconjugant cfu were plated on LB agar with gentamycin, incubated at

$75737^{\circ} \mathrm{C}$ overnight, scraped from agar into liquid LB medium, diluted to $\mathrm{OD}_{600}=0.25$ into $10 \mathrm{~mL}$ LB

758 plus gentamycin in $50 \mathrm{~mL}$ conical tubes, incubated at $37^{\circ} \mathrm{C} 250 \mathrm{rpm}$ until $\mathrm{OD}_{600}=1.0$, centrifuged

759 at $4,000 \mathrm{~g}$, and frozen for downstream analysis. To determine the percent of selectable transposed

760 colonies possessing on-target and off-target edits, the total number of selectable colonies was

761 adjusted (Extended Data Fig. 4b) for on-target and off-target percent as determined by ET-Seq

762 (Fig. 4b). ET-Seq analysis was conducted on triplicate platings of DART transconjugants $(n=3$

763 for each system) to identify transposon insertion locations and quantify on-target vs. off-target

764 insertions. As the targeted genomic region encoding the lacZ a-peptide is duplicated in E. coli

765 BL21(DE3), one of the two duplicated regions $(749,903 \mathrm{bp}-->750,380 \mathrm{bp})$ was removed prior to

766 analysis to allow unambiguous mapping assignment. Subsequently, the standard ETsuite

767 analysis pipeline (as described above) was used to identify and map $300 \mathrm{bp} \times 2$ reads containing

768 transposon junctions back to the recipient BL21(DE3) genome and cluster barcodes that

769 corresponded to unique insertion events. To confirm an insert location we first identified the exact

770 transposon-genome junction mapping coordinate that was the most frequent in the reads of a

771 barcode cluster (prime location) then required that a barcode cluster had: (1) at least $75 \%$ of its

772 reads coming from within $3 \mathrm{bp}$ of the prime location and (2) at least $75 \%$ of its reads mapping to

773 the same strand. If these criteria were true the barcode cluster was counted as a unique insertion

774 and the prime location was used as the mapping locus by ET-Seq. An on-target insertion was

775 evaluated as a barcode cluster with a prime location within 200 bp downstream of the 3' end of 
776 the protospacer target. Finally all distances reported from the protospacer target site were

777 calculated from the last base pair of the 3' end of the protospacer.

778

779 VcDART-mediated targeted editing in a community.

780 VcDART vectors encoding constitutive VcCasTn, constitutive bla:aadA Tn cargo (2.7 kbp), and

781 either a non-targeting (pBFC0888), K. michiganensis M5al pyrF-targeting (pBFC0825), or $P$.

782 simiae WCS417 pyrF-targeting (pBFC0837) constitutive crRNA were transformed into E. coli

783 WM3064. Conjugations of these vectors into the nine-member community were performed as

784 described above on filter-topped LB agar plates with $12 \mathrm{hr}$ incubation at $30^{\circ} \mathrm{C}$. Lawns were

785 scraped from filters into $10 \mathrm{~mL}$ LB medium, vortexed, and $1 \mathrm{OD}_{600}{ }^{*} \mathrm{~mL}$ from each lawn was plated

786 on LB agar supplemented with $1 \mathrm{mg} \mathrm{mL}^{-1} 5$-FOA, $100 \mu \mathrm{g} \mathrm{mL}-1$ carbenicillin, $100 \mu \mathrm{mL}^{-1}$

787 streptomycin, and $100 \mu \mathrm{g} \mathrm{mL} \mathrm{m}^{-1}$ spectinomycin. Following 3 days of incubation at $30^{\circ} \mathrm{C}$, all cells

788 were scraped from the agar into $10 \mathrm{~mL}$ R2A medium, vortexed, diluted into $10 \mathrm{~mL} R 2 \mathrm{~A}$

789 supplemented with $20 \mathrm{mg} \mathrm{mL}^{-1}$ uracil (for no selection controls) or R2A with uracil, 5-FOA,

790 carbenicillin, streptomycin, and spectinomycin to $\mathrm{OD}_{600}=0.02$, and split evenly across 4 wells

791 (2.5 mL/well) of a 24 deep well plate. After cultivation at $30^{\circ} \mathrm{C}$ and $750 \mathrm{rpm}$ for 1 week, only the

792 cultures conjugated with VcDART containing pyrF-targeting crRNA had grown in presence of

793 antibiotics and 5-FOA. A small portion of each of these cultures was serially diluted in R2A and

794 plated on LB agar plus antibiotics to isolate and assay colonies by targeted PCR and Sanger

795 sequencing of $p y r F$ loci. The remainder of each culture was centrifuged at $4,000 \mathrm{~g}$ for $10 \mathrm{~min}$ and

796 frozen at $-80^{\circ} \mathrm{C}$ for downstream bacterial $16 \mathrm{~S}$ rRNA V4 amplicon metagenomic sequencing

797 (Novogene). Relative abundances were calculated as described below (16S rRNA V4 amplicon

798 analysis) for pre-conjugation nine-member community cultures and post-selection pyrF-targeted

799 cultures.

800

801 16S rRNA V4 amplicon analysis 
16S rRNA V4 amplicon sequencing was conducted using the 515F (5'GTGCCAGCMGCCGCGGTAA-3') and 806R (5'-GGACTACHVGGGTWTCTAAT-3') universal

804 bacterial primer set to generate 250 bp x 2 reads (Novogene). Samples were processed using 805 the UPARSE pipeline within the USEARCH software package to merge read pairs, remove 806 primers, quality filter sequences, remove chimeras, identify unique sequence variants (ZOTUs),

807 and quantify their abundance across samples as described previously ${ }^{24}$. To assign ZOTU 808 sequences to species known to be in our community mixture, we queried all 890 identified ZOTUs 809 against a custom database of $16 \mathrm{~S}$ sequences derived from the genomes of the nine-member 810 community constituents using USEARCH ${ }^{25}$ ZOTUs with $100 \%$ identity to a $16 \mathrm{~S}$ sequence in our 811 database were assigned to the matched species, and all matches $<100 \%$ identity were counted

812 as "Other". Counts coming from ZOTUs of the same taxonomic assignment were merged and the 813 relative abundance of a species was calculated as its read counts divided by the total read counts 814 for the sample.

816 Statistics and reproducibility

817 All transformations (natural transformation, conjugation, electroporation) and subsequent 818 analyses were performed for three independent replicates.

820 Reporting summary

821 Further information on research design is available in the Nature Research Reporting Summary 822 linked to this paper.

\section{Data availability}

825 Summary data for genomes, plasmids, and oligonucleotides used in this study can be found in

826 Supplementary tables 1-4. Sequence data for all genomes assembled as part of this study and 827 newly constructed plasmids are in submission to NCBI with accession numbers pending. 
828 Sequence data for genomes taken from Huddy, et $\mathrm{al}^{8}$ are in submission to NCBI with accession

829 numbers pending. Sequence data for genomes taken from Kantor, et al ${ }^{9}$ are available under $\mathrm{NCBI}$

830 BioProject accession no. PRJNA279279. All genomes and plasmids used in the project will also

831 be made available on ggKbase (https://ggkbase.berkeley.edu/). Raw count data for all

832 experiments including both metagenome and ET-seq information is available at

833 https://github.com/SDmetagenomics/ETsuite/tree/master/manuscript data.

835 Code availability

836 Custom R scripts for ET-Seq analysis and code used in the construction of figures are available

837 at https://github.com/SDmetagenomics/ETsuite.

\section{Methods references}

840 1. Adler, B. A. et al. Systematic Discovery of Salmonella Phage-Host Interactions via High-

$841 \quad$ Throughput Genome-Wide Screens. doi:10.1101/2020.04.27.058388.

842 2. Klompe, S. E., Vo, P. L. H., Halpin-Healy, T. S. \& Sternberg, S. H. Transposon-encoded

843 CRISPR-Cas systems direct RNA-guided DNA integration. Nature 571, 219-225 (2019).

844 3. Strecker, J. et al. RNA-guided DNA insertion with CRISPR-associated transposases.

$845 \quad$ Science 365, 48-53 (2019).

846 4. Liu, H. et al. Magic Pools: Parallel Assessment of Transposon Delivery Vectors in Bacteria.

847 mSystems 3, (2018).

848 5. Liu, H. et al. Large-scale chemical-genetics of the human gut bacterium Bacteroides

849 thetaiotaomicron. BioRxiv (2019).

850 6. Devon, R. S., Porteous, D. J. \& Brookes, A. J. Splinkerettes--improved vectorettes for

851 greater efficiency in PCR walking. Nucleic Acids Res. 23, 1644-1645 (1995).

852 7. Barquist, L. et al. The TraDIS toolkit: sequencing and analysis for dense transposon mutant 
libraries. Bioinformatics 32, 1109-1111 (2016).

854 8. Huddy, R. J. et al. Thiocyanate and organic carbon inputs drive convergent selection for specific autotrophic Afipia and Thiobacillus strains within complex microbiomes. bioRxiv 2020.04.29.067207 (2020) doi:10.1101/2020.04.29.067207.

9. Kantor, R. S. et al. Genome-Resolved Meta-Omics Ties Microbial Dynamics to Process

10. Olm, M. R., Brown, C. T., Brooks, B. \& Banfield, J. F. dRep: a tool for fast and accurate genomic comparisons that enables improved genome recovery from metagenomes through de-replication. ISME J. 11, 2864-2868 (2017).

11. Parks, D. H., Imelfort, M., Skennerton, C. T., Hugenholtz, P. \& Tyson, G. W. CheckM: assessing the quality of microbial genomes recovered from isolates, single cells, and

12. Chaumeil, P.-A., Mussig, A. J., Hugenholtz, P. \& Parks, D. H. GTDB-Tk: a toolkit to classify genomes with the Genome Taxonomy Database. Bioinformatics (2019) doi:10.1093/bioinformatics/btz848.

13. Diamond, S. et al. Mediterranean grassland soil C-N compound turnover is dependent on rainfall and depth, and is mediated by genomically divergent microorganisms. Nat Microbiol

872 14. Edgar, R. C. MUSCLE: multiple sequence alignment with high accuracy and high 873 throughput. Nucleic Acids Res. 32, 1792-1797 (2004).

874 15. Nguyen, L.-T., Schmidt, H. A., von Haeseler, A. \& Minh, B. Q. IQ-TREE: a fast and effective 875 stochastic algorithm for estimating maximum-likelihood phylogenies. Mol. Biol. Evol. 32, $876 \quad 268-274(2015)$.

877 16. Peng, Y., Leung, H. C. M., Yiu, S. M. \& Chin, F. Y. L. IDBA-UD: a de novo assembler for 878 single-cell and metagenomic sequencing data with highly uneven depth. Bioinformatics 28, 
$1420-1428$ (2012).

17. Hyatt, D. et al. Prodigal: prokaryotic gene recognition and translation initiation site identification. BMC Bioinformatics 11, 119 (2010).

18. Lowe, T. M. \& Eddy, S. R. tRNAscan-SE: a program for improved detection of transfer RNA genes in genomic sequence. Nucleic Acids Res. 25, 955-964 (1997).

19. Martin, M. Cutadapt removes adapter sequences from high-throughput sequencing reads. EMBnet.journal 17, 10-12 (2011).

20. Langmead, B. \& Salzberg, S. L. Fast gapped-read alignment with Bowtie 2. Nat. Methods

21. Zhao, L., Liu, Z., Levy, S. F. \& Wu, S. Bartender: a fast and accurate clustering algorithm to count barcode reads. Bioinformatics 34, 739-747 (2018).

22. Costello, M. et al. Characterization and remediation of sample index swaps by nonredundant dual indexing on massively parallel sequencing platforms. BMC Genomics 19, 332 (2018).

23. R Core Team. R: A language and environment for statistical computing. (2013).

24. Edgar, R. C. UPARSE: highly accurate OTU sequences from microbial amplicon reads.

\section{Acknowledgments}

900 We thank Morgan N. Price for data analysis input, Patrick Pausch for experimental advice, Shana

901 L. McDevitt, Eileen Wagner, and Hitomi Asahara for help with sequencing, and Trent R. Northen 902 for directional advice. Funding was provided by m-CAFEs Microbial Community Analysis \& 903 Functional Evaluation in Soils, (m-CAFEs@lbl.gov) a project led by Lawrence Berkeley National 
904 Laboratory supported by the U.S. Department of Energy, Office of Science, Office of Biological \&

905 Environmental Research under contract number DE-AC02-05CH11231. Support was also

906 provided by the Innovative Genomics Institute at UC Berkeley. B.E.R. and B.F.C. are supported

907 by the National Institute of General Medical Sciences of the National Institute of Health under

908 award numbers F32GM134694 and F32GM131654. Schematics were created with

909 BioRender.com.

910

911 Contributions

912 B.E.R., S.D., B.F.C, A.M.D., J.F.B, and J.A.D. conceived the work and designed the experiments.

913 B.E.R., B.F.C., C.H., M.X., Z.Z., D.C.S., K.T., T.K.O., and N.K. conducted the molecular biology

914 included. S.D., A.C.-C., C.H., and R.S. developed the bioinformatic analysis. B.E.R., S.D., B.F.C.,

915 A.M.D., J.F.B., and J.A.D. analyzed and interpreted the data.

916

917 Competing Interests

918 The Regents of the University of California have patents pending related to this work on which

919 B.E.R., S.D., B.F.C., A.M.D., J.F.B., and J.A.D. are inventors. J.A.D. is a co-founder of Caribou

920 Biosciences, Editas Medicine, Intellia Therapeutics, Scribe Therapeutics and Mammoth

921 Biosciences, a scientific advisory board member of Caribou Biosciences, Intellia Therapeutics,

922 eFFECTOR Therapeutics, Scribe Therapeutics, Synthego, Mammoth Biosciences and Inari, and

923 is a Director at Johnson \& Johnson and has sponsored research projects by Biogen, Roche and

924 Pfizer. J.F.B. is a founder of Metagenomi.

925

926 Additional Information

927 Correspondence and request for materials should be addressed to J.A.D. and J.F.B. 


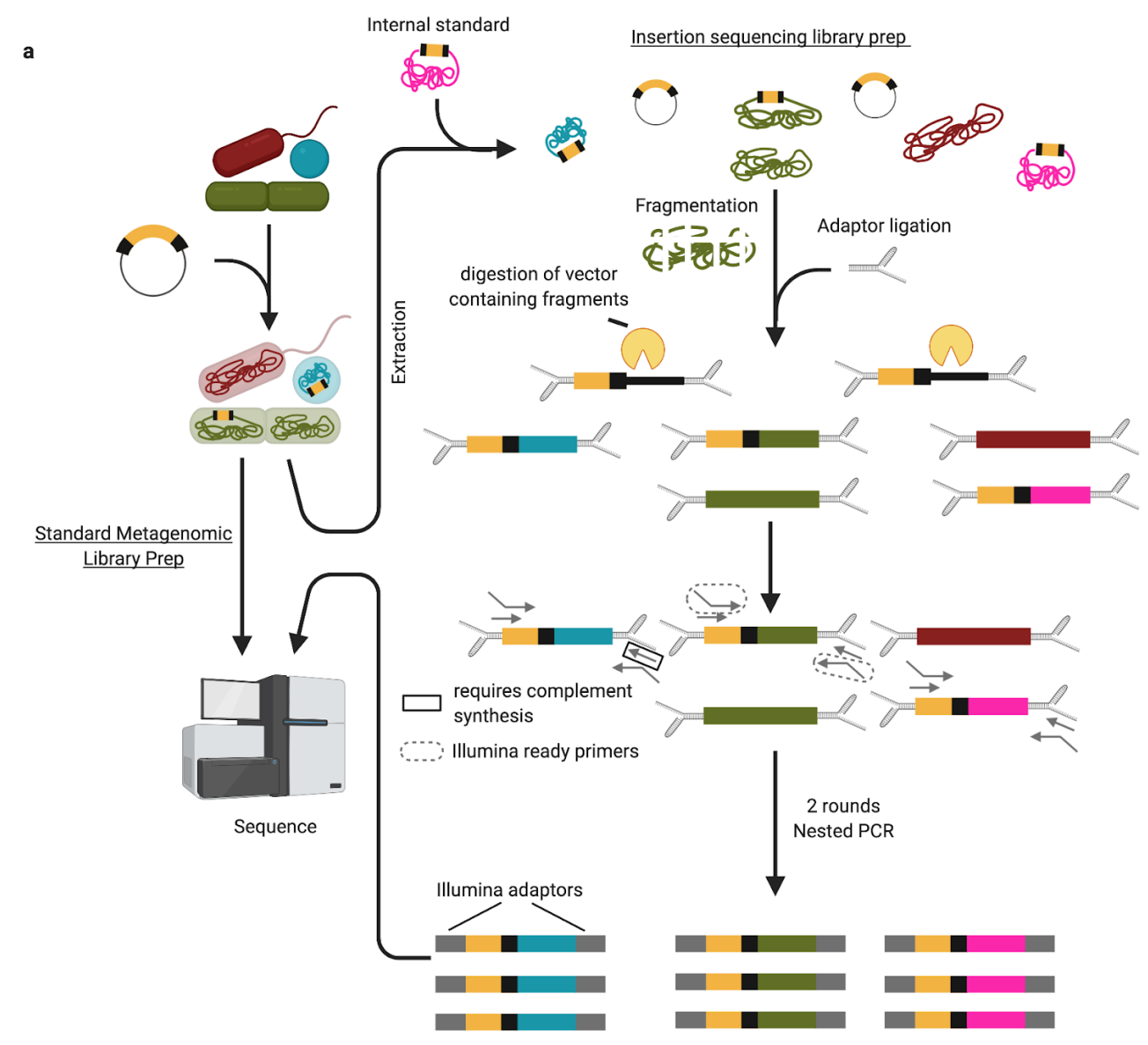

b
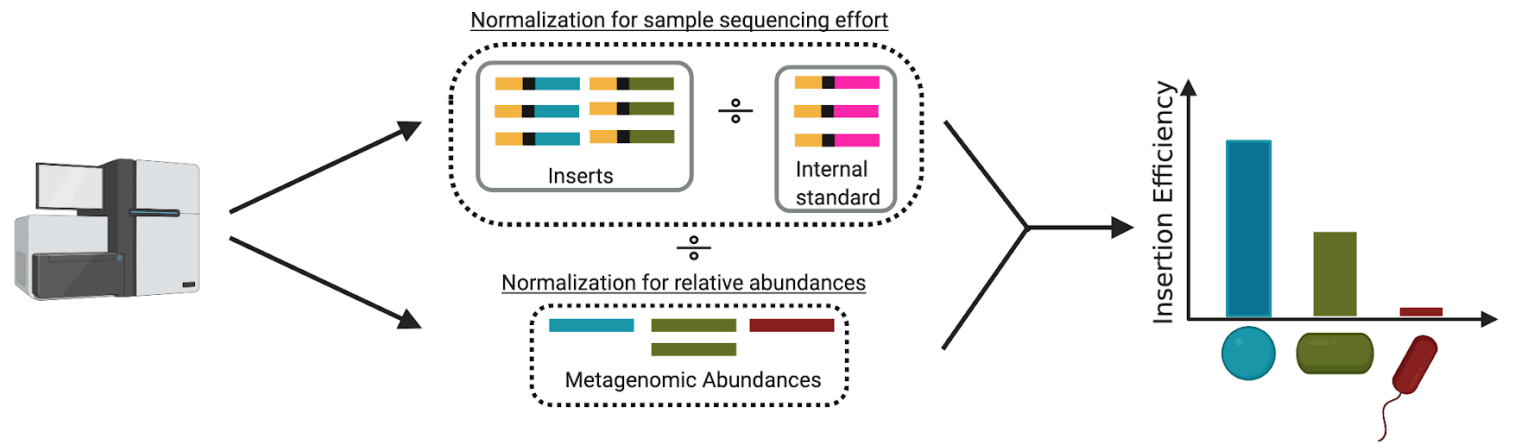

930 Extended Data Fig. 1 | Library preparation and data normalization for ET-Seq. a, ET-Seq requires low-

931 coverage metagenomic sequencing and customized insertion sequencing. Insertion sequencing relies on custom splinkerette adaptors, which minimize non-specific amplification, a digestion step for degradation

933 of delivery vector containing fragments, and nested PCR to enrich for fragments containing insertions with 
bioRxiv preprint doi: https://doi.org/10.1101/2020.07.17.209189; this version posted July 21, 2020. The copyright holder for this preprint (which was not certified by peer review) is the author/funder, who has granted bioRxiv a license to display the preprint in perpetuity. It is made available under aCC-BY-NC 4.0 International license.

934 high specificity. The second round of nested PCR adds unique dual index adaptors for Illumina sequencing.

935 b, This insertion sequencing data is first normalized by the reads to internal standard DNA which is added

936 equally to all samples and serves to correct for variation in reads produced per sample. Secondly, it is 937 normalized by the relative metagenomic abundances of the community members. 

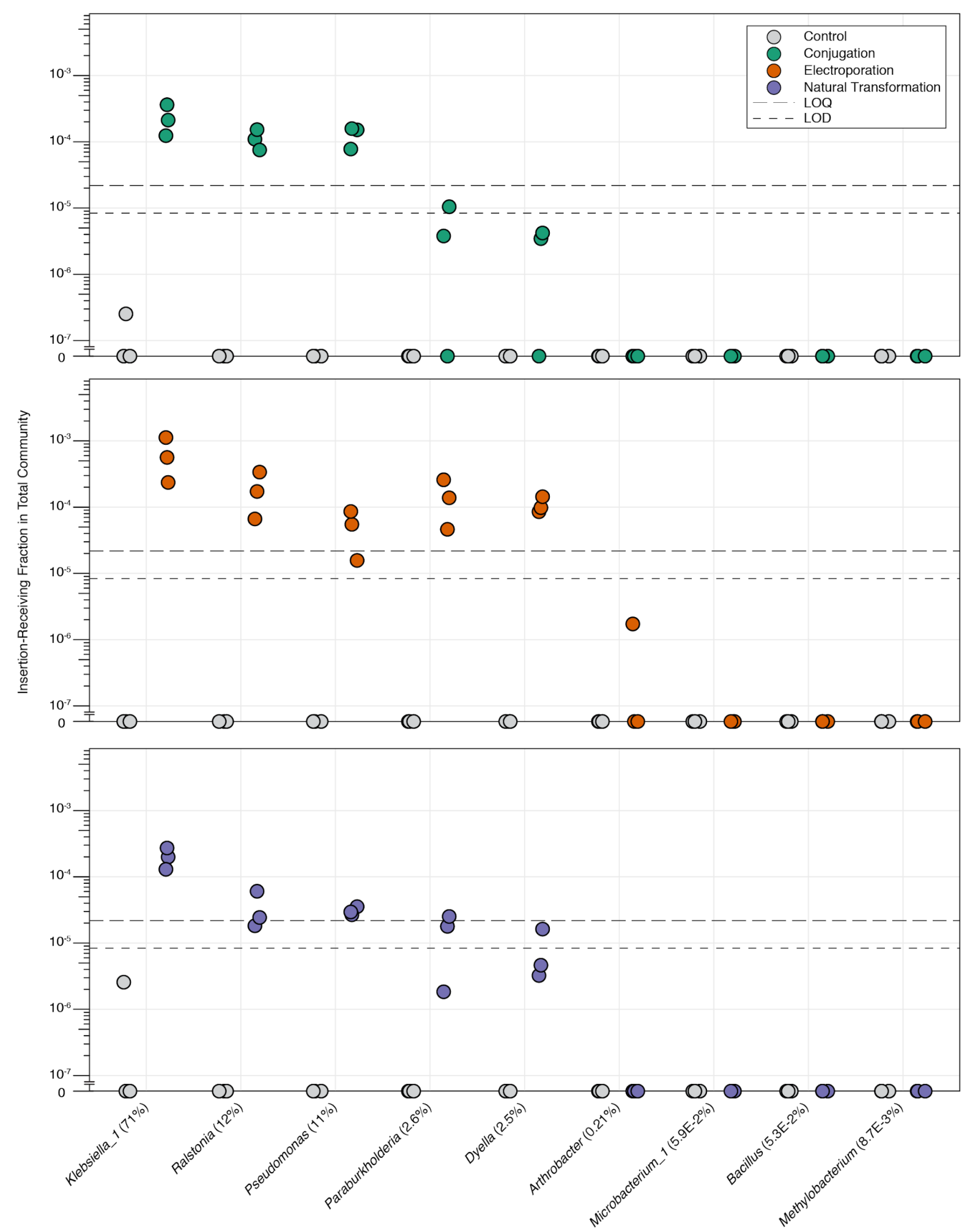

939 Extended Data Fig. 2 | ET-Seq determined insertion efficiencies for all nine consortium members as

940 a fraction of the entire community. ET-Seq determined insertion efficiencies for conjugation, 
bioRxiv preprint doi: https://doi.org/10.1101/2020.07.17.209189; this version posted July 21, 2020. The copyright holder for this preprint (which was not certified by peer review) is the author/funder, who has granted bioRxiv a license to display the preprint in perpetuity. It is made available under aCC-BY-NC 4.0 International license.

941 electroporation, and natural transformation on the nine-member synthetic community $(n=3$ biological

942 replicates). The values shown are the estimated fraction a constituent species's transformed cells make of

943 the total community population. Control samples received no exogenous DNA. Average relative abundance

944 across all samples is indicated in parentheses ( $n=18$ independent samples). LOD and LOQ are indicated

945 in plots by short and long dashed lines respectively.

946 

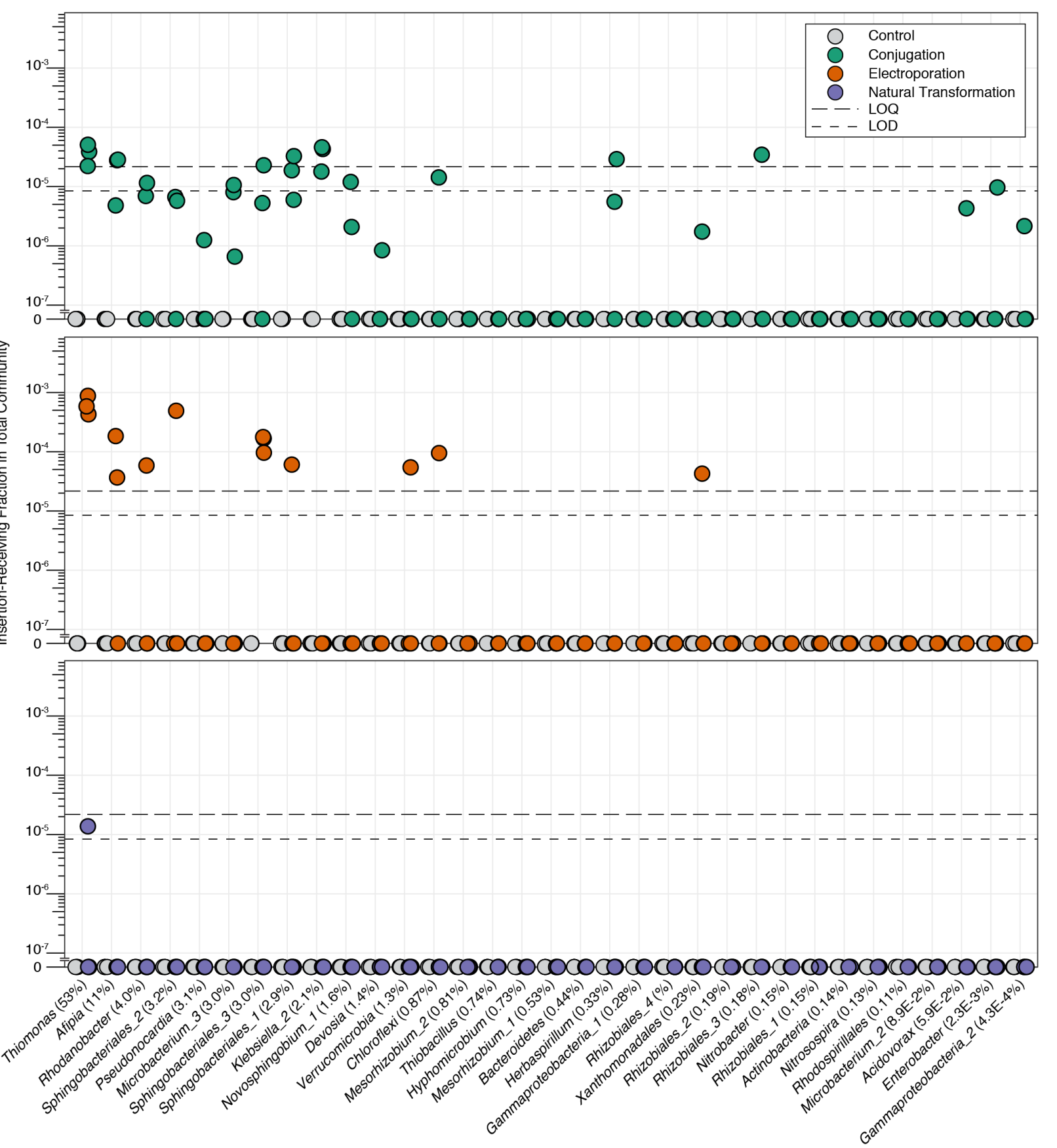

949 Extended Data Fig. 3 | ET-Seq determined insertion efficiencies for all thiocyanate-degrading

950 bioreactor community members as a fraction of the entire community. ET-Seq determined insertion

951 efficiencies for conjugation, electroporation, and natural transformation on the thiocyanate-degrading

952 bioreactor community ( $n=3$ biological replicates). The values shown are the estimated fraction a

953 constituent species's transformed cells make of the total community population. Control samples received 
bioRxiv preprint doi: https://doi.org/10.1101/2020.07.17.209189; this version posted July 21, 2020. The copyright holder for this preprint (which was not certified by peer review) is the author/funder, who has granted bioRxiv a license to display the preprint in perpetuity. It is made available under aCC-BY-NC 4.0 International license.

954 no exogenous DNA. Average relative abundance across all samples is indicated in parentheses $(n=17$

955 independent samples; due to a single failed metagenomic sequencing replicate, see methods). LOD and

956 LOQ are indicated in plots by short and long dashed lines respectively. 
bioRxiv preprint doi: https://doi.org/10.1101/2020.07.17.209189; this version posted July 21, 2020. The copyright holder for this preprint (which was not certified by peer review) is the author/funder, who has granted bioRxiv a license to display the preprint in perpetuity. It is made available under aCC-BY-NC 4.0 International license.

a

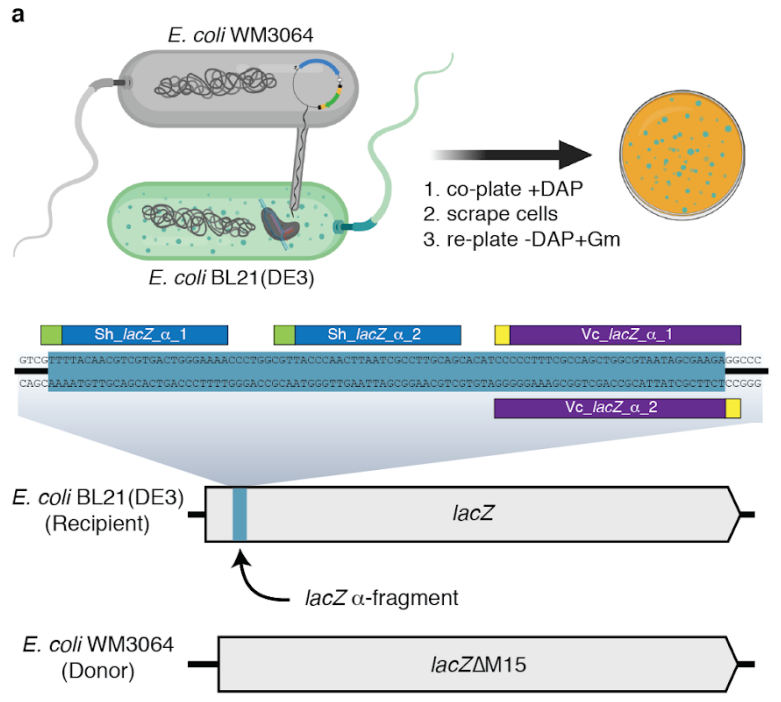

c

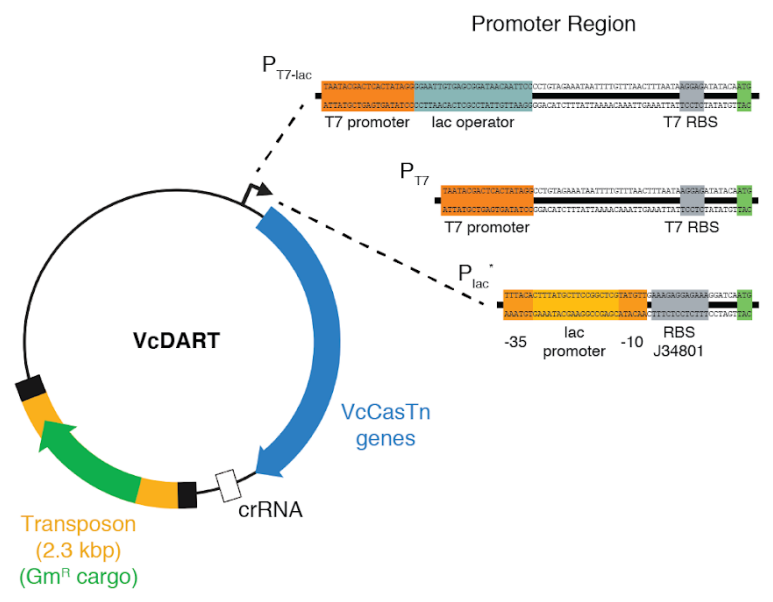

e

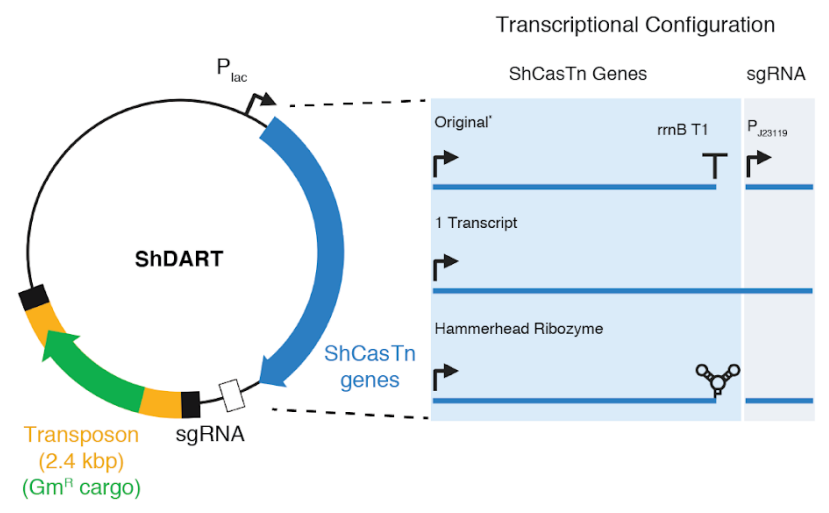

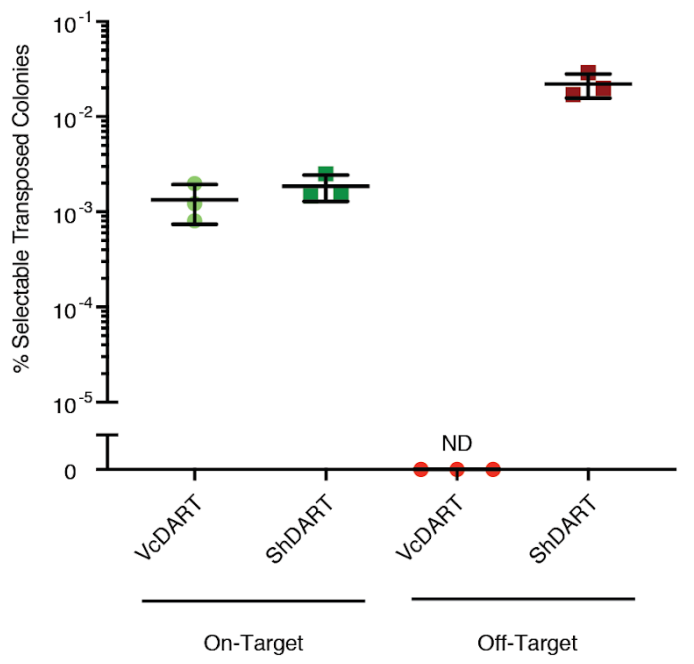

d
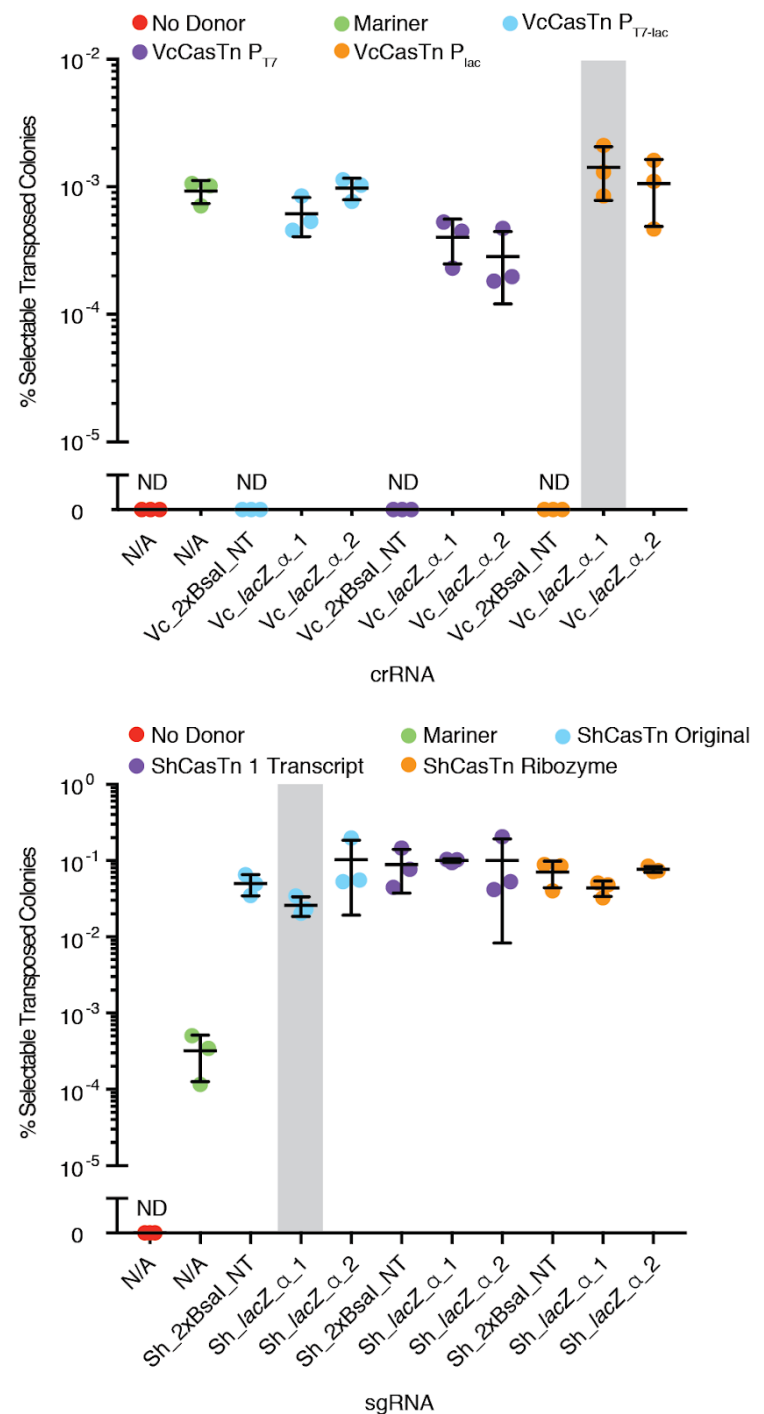
958 Extended Data Fig. 4 | Benchmarking DART vectors. a, E. coli WM3064 to E. coli BL21(DE3) conjugation,

959 transposition, and selection schematic (top) and guide RNAs targeting the lacZ a-fragment of recipient BL21(DE3),

960 which is absent from donor WM3064 (bottom). b,d,f, Percent selectable transposed colonies is calculated as the

961 number of colonies obtained with gentamycin selection divided by total viable colonies in absence of selection. $\mathbf{b}$,

962 Insertion receiving colonies divided into on- and off-targeted. This was calculated by multiplying \% selectable colonies

963 for representative guides in $\mathbf{d}$ and $\mathbf{f}$ (highlighted by grey bars) by the on- or off-target rates (shown in Fig. 4). c,

964 Transposition with VcDART was tested with three promoters. The variant using the Plac promoter, harvested from

965 pHelper_ShCAST_sgRNA ${ }^{18}$, was also used for Fig. 4, 5, and Extended Data Fig. 4b (*). d, Efficiencies of VcDART

966 using various promoters. e, Transposition with ShDART was tested with three transcriptional configurations, all using

$967 \mathrm{P}_{\mathrm{lac}}{ }^{18}$. The configuration used for characterization of ShCasTn originally ${ }^{18}$ was also used for Fig. 4 and Extended Data

968 Fig. 4b $\left(^{*}\right)$. f, Efficiencies of ShDART using various promoters. b, d, f, Crossbar indicates mean and error bars indicate

969 one standard deviation from the mean ( $n=3$ biological replicates). Guide RNAs ending in "NT" are non-targeting

970 negative control samples. 